\title{
REPRESENTATIONS ASSOCIATED TO SMALL NILPOTENT ORBITS FOR COMPLEX SPIN GROUPS
}

\author{
DAN BARBASCH AND WAN-YU TSAI
}

\begin{abstract}
This paper provides a comparison between the $K$-structure of unipotent representations and regular sections of bundles on nilpotent orbits for complex groups of type $D$. Precisely, let $G_{0}=\operatorname{Spin}(2 n, \mathbb{C})$ be the Spin complex group as a real group, and let $K \cong G_{0}$ be the complexification of the maximal compact subgroup of $G_{0}$. We compute $K$-spectra of the regular functions on some small nilpotent orbits $\mathcal{O}$ transforming according to characters $\psi$ of $C_{K}(\mathcal{O})$ trivial on the connected component of the identity $C_{K}(\mathcal{O})^{0}$. We then match them with the $K$-types of the genuine (i.e., representations which do not factor to $\mathrm{SO}(2 n, \mathbb{C}))$ unipotent representations attached to $\mathcal{O}$.
\end{abstract}

\section{INTRODUCTION}

Let $G$ be a connected complex semisimple group (viewed as a real group), and let $\mathfrak{g}, \mathfrak{g}^{\vee}, \mathfrak{g}_{\mathbb{C}}$ be its Lie algebra, dual Lie algebra, and complexified Lie algebra, respectively. Special unipotent representations of $G$ were introduced in [BV85]. To each nilpotent orbit $\mathcal{O}^{\vee} \subset \mathfrak{g}^{\vee}$ an infinitesimal character $\lambda_{\mathcal{O}} \vee$ is associated via the Jacobson-Morozov theorem; the orbit is associated to a Lie triple $\left\{e^{\vee}, h^{\vee}, f^{\vee}\right\}$, and $\lambda_{\mathcal{O}^{\vee}}=h^{\vee} / 2$ determines an infinitesimal character. Special unipotent representations are defined as the irreducible $\left(\mathfrak{g}_{\mathbb{C}}, K\right)$-modules $\Xi$ satisfying

(1) the infinitesimal character is $\left(\lambda_{\mathcal{O}} \vee, \lambda_{\mathcal{O}} \vee\right)$ (see Section 4 for the parametrization of $\left(\mathfrak{g}_{\mathbb{C}}, K\right)$-modules) and

(2) the annihilator of $\Xi$ in the universal enveloping algebra $U\left(\mathfrak{g}_{\mathbb{C}}\right)$, denoted $A n n_{U\left(\mathfrak{g}_{\mathbb{C}}\right)} \Xi$, is the unique maximal primitive ideal of $U\left(\mathfrak{g}_{\mathbb{C}}\right)$ with infinitesimal character $\left(\lambda_{\mathcal{O}}, \lambda_{\mathcal{O}}\right)$.

Denote by $\mathcal{U}_{G}\left(\mathcal{O}^{\vee}, \lambda_{\mathcal{O}} \vee\right)$ the set of unipotent representations of $G$ associated to $\mathcal{O}^{\vee}$. In Bar89, the unitarity of these representations is established for the case of classical groups, and the whole unitary dual for such groups is determined. In the process, a larger set of representations is introduced which are called unipotent. A finite set of infinitesimal characters $\lambda_{\mathcal{O}^{\vee}, s^{\vee}}$ is associated to each $\mathcal{O}^{\vee}$, and an irreducible module $\Xi$ is unipotent if it satisfies (1) and (2) with the more general $\lambda_{\mathcal{O} \vee, s} \vee$ instead. The results in Bar89 can be viewed as proving that the modules in $\mathcal{U}_{G}\left(\mathcal{O}^{\vee}, \lambda_{\mathcal{O}^{\vee}, s^{\vee}}\right)$ are the building blocks of the unitary dual.

In [Bar17] a different viewpoint is taken. Instead of parametrizing by $\mathcal{O}^{\vee}$, the unipotent representations are parametrized by nilpotent orbits $\mathcal{O} \subset \mathfrak{g}$. The precise setting is as follows. Let $G_{0} \subset G$ be the real points of a complex linear reductive

\footnotetext{
Received by the editors September 5, 2017, and, in revised form, April 2, 2018.

2010 Mathematics Subject Classification. Primary 22E46, 22E47.

Key words and phrases. Infinte dimensional representations, orthogonal groups, nilpotent orbits.

The first author was supported in part by NSA Grant H98230-16-1-0006.
} 
algebraic group $G$ with Lie algebra $\mathfrak{g}_{0}$ and maximal compact subgroup $K_{0}$. Let $\mathfrak{g}_{0}=\mathfrak{k}_{0}+\mathfrak{s}_{0}$ be the Cartan decomposition, and let $\mathfrak{g}=\mathfrak{k}+\mathfrak{s}$ be the complexification. Let $K$ be the complexification of $K_{0}$. Then $\mathrm{K}$ acts on $\mathfrak{s}$ by the adjoint action. We denote by $C_{K}(e)=\{g \in K \mid A d(g) e=e\}$ the centralizer of $e \in \mathfrak{s}$ in $K$.

For each irreducible admissible representation $\Xi$ of $G_{0}$, Vog91 defines the associated cycle $A C(\Xi)$ to be a union of nilpotent $K$-orbits in $\mathfrak{s}$, and an algebraic representation of each centralizer $C_{K}(e)$ of a representative $e$ for each orbit in $A C(\Xi)$.

Assume that $G_{0}$ is a connected complex group viewed as a real Lie group. Then $G \cong G_{0} \times G_{0}$, and $K \cong G_{0}$ as complex groups. Furthermore $\mathfrak{s} \cong \mathfrak{g}_{0}$ as complex vector spaces. In this case there is only one $K$-orbit $\mathcal{O} \subset \mathfrak{s} \cong \mathfrak{g}_{0}$ in $A C(\Xi)$. The main results in [Vog00, Chapter 7] and [Vog91, Theorem 4.11] imply that, in the case of a complex group,

$$
\left.\Xi\right|_{K}=R(\mathcal{O}, \psi)-Y
$$

with $\psi$ an algebraic representation, $R(\mathcal{O}, \psi)$ as defined in equation (1.1.1) below, and $Y$ an $S(\mathfrak{g} / \mathfrak{k})$-module supported on orbits of strictly smaller dimension.

Definition 1.1. Let $e \in \mathfrak{s}$ be a nilpotent element, and let $\mathcal{O}:=K \cdot e \subset \mathfrak{s}$ be the $K$-adjoint orbit of $e$. We say that an irreducible admissible representation $\Xi$ is associated to $\mathcal{O}$ if $\mathcal{O}$ occurs with nonzero multiplicity in the associated cycle. We will write $\mathcal{U}_{G_{0}}(\mathcal{O}, \lambda)$ for the set of irreducible representations of $G_{0}$ with maximal annihilator with infinitesimal character $\lambda$, and associated to $\mathcal{O}$.

Let $C_{K}(\mathcal{O}):=C_{K}(e)$ denote the centralizer of $e$ in $K$, with $e$ a representative of $\mathcal{O}$, and let $A_{K}(\mathcal{O}):=C_{K}(\mathcal{O}) / C_{K}(\mathcal{O})^{0}$ be the component group. In the case of $G_{0}$ being a connected complex group viewed as a real group, it is conjectured that there exists an infinitesimal character $\lambda_{\mathcal{O}}$ such that in addition, we have the following.

Conjecture. For each $\mathcal{O}$ there is $\lambda_{\mathcal{O}}$ such that there is a 1-1 correspondence

$$
\psi \in \widehat{A_{K}(\mathcal{O})} \longleftrightarrow \Xi(\mathcal{O}, \psi) \in \mathcal{U}_{G_{0}}\left(\mathcal{O}, \lambda_{\mathcal{O}}\right)
$$

satisfying the additional condition

$$
\left.\Xi(\mathcal{O}, \psi)\right|_{K} \cong R(\mathcal{O}, \psi),
$$

where

$$
\begin{aligned}
R(\mathcal{O}, \psi) & =\operatorname{Ind}_{C_{K}(\mathcal{O})}^{K}(\psi) \\
& =\left\{f: K \rightarrow V_{\psi} \mid f(g x)=\psi\left(x^{-1}\right) f(g) \forall g \in K, x \in C_{K}(\mathcal{O})\right\}
\end{aligned}
$$

is the ring of regular functions on $\mathcal{O}$ transforming according to $\psi$.

It is also natural to conjecture that there is a choice of $\lambda_{\mathcal{O}}$ such that the representations are unitary. The results in Bar89 and Bre99 establish the unitarity of the modules considered in this paper.

Candidates for parameters $\lambda_{\mathcal{O}}$ satisfying the Conjecture above are listed in [Bar17. Essentially they coincide with the $\lambda_{\mathcal{O}^{\vee}, s^{\vee}}$ introduced in Bar89]. The validity of the conjecture is established for large classes of nilpotent orbits in the classical complex groups. Such parameters $\lambda_{\mathcal{O}}$ are available for the exceptional groups as well, Bar17] for $F_{4}$, and to appear elsewhere for type $E$. 
This conjecture cannot be valid for all nilpotent orbits in the case of real groups; $A C(\Xi)$ is supported on several $K$-orbits which are the components of the intersection of a complex nilpotent orbit $\mathcal{O}_{c}$ with $\mathfrak{s}$. The $R(\mathcal{O}, \psi)$ can be the same for different components of a particular $\mathcal{O}_{c}$. The representations with associated cycle containing a given component have drastically different $K$-structures. Examples can be found in $\operatorname{Vog} 00$. In addition, many examples are known (e.g., the case of the minimal orbit in certain real forms of type $D$ ) for which there are no representations with associated cycle supported on $\mathcal{O}$ or any real form of its complexification. The analogues of the results in this paper in the case of the real Spin groups are studied in BT18].

In the case when $\psi$ is the trivial representation, our results provide irreducible (unitary) $(\mathfrak{g}, K)$-modules with $K$-structure $R(\mathcal{O})$. By the Kraft-Procesi classification [KP82 of nilpotent orbits whose closures are normal, the orbits we consider are normal, so also $R(\mathcal{O})=R(\overline{\mathcal{O}})$.

In this paper we investigate this conjecture for small orbits in the complex case using different techniques than in Bar17]; [BT18] investigates the analogue for the real Spin groups. For the condition of small we require that

$$
[\mu: R(\mathcal{O}, \psi)] \leq c_{\mathcal{O}}
$$

i.e., that the multiplicity of any $\mu \in \widehat{K}$ be uniformly bounded. This puts a restriction on $\operatorname{dim} \mathcal{O}$ :

$$
\operatorname{dim} \mathcal{O} \leq \operatorname{rank}(\mathfrak{k})+\left|\Delta^{+}(\mathfrak{k}, \mathfrak{t})\right|,
$$

where $\mathfrak{t} \subset \mathfrak{k}$ is a Cartan subalgebra and $\Delta^{+}(\mathfrak{k}, \mathfrak{t})$ is a positive system. The reason for this restriction is as follows. Let $(\Pi, X)$ be an admissible representation of $G_{0}$, and let $\mu$ be the highest weight of a representation $(\pi, V) \in \widehat{K}$ which is dominant for $\Delta^{+}(\mathfrak{k}, \mathfrak{t})$. Assume that $\operatorname{dim}_{\operatorname{Hom}_{K}}[\pi, \Pi] \leq C$ and $\Pi$ has associated variety (cf. [Vog91]). Then

$\operatorname{dim}\{v: v \in X$ belongs to an isotypic component with $\|\mu\| \leq t\} \leq C t^{\left|\Delta^{+}(\mathfrak{k}, \mathfrak{t})\right|+\operatorname{dim} \mathfrak{t}}$.

The dimension of $(\pi, V)$ grows like $t^{\left|\Delta^{+}(\mathfrak{k}, \mathfrak{t})\right|}$, the number of representations with highest weight $\|\mu\| \leq t$ grows like $t^{\operatorname{dim} t}$, and the multiplicities are assumed uniformly bounded. On the other hand, considerations involving primitive ideals imply that the dimension of this set grows like $t^{\operatorname{dim} G \cdot e / 2}$ with $e \in \mathcal{O}$, and half the dimension of (the complex orbit) $G \cdot e$ is the dimension of the (K-orbit) $K \cdot e \in \mathfrak{s}$. In the case of type $D$, condition (1.1.2) coincides with being spherical; see Pan94. Since we only deal with characters of $C_{K}(\mathcal{O})$, multiplicity $\leq 1$ is guaranteed.

In the case of the complex groups of type $D_{n}$, we consider $G_{0}=\operatorname{Spin}(2 n, \mathbb{C})$ viewed as a real group, and hence $K \cong G_{0}$ is the complexification of the maximal compact subgroup $K_{0}=\operatorname{Spin}(2 n)$ of $G$. In Section 2 we list all small nilpotent orbits satisfying (1.1.2) and describe (the component groups of) their centralizers. In Section 3, we compute $R(\mathcal{O}, \psi)$ for each $\mathcal{O}$ in Subsection 2.1 and $\psi \in \widehat{A_{K}(\mathcal{O})}$. In Section 4 we associate to each $\mathcal{O}$ an infinitesimal character $\lambda_{\mathcal{O}}$ by Bar17. The fact is that $\mathcal{O}$ is the minimal orbit which can be the associated variety of a $(\mathfrak{g}, K)$ module with infinitesimal character $\left(\lambda_{L}, \lambda_{R}\right)$, with $\lambda_{L}$ and $\lambda_{R}$ both conjugate to $\lambda_{\mathcal{O}}$. We make a complete list of irreducible modules $\bar{X}\left(\lambda_{L}, \lambda_{R}\right)$ (in terms of Langlands classification) which are attached to $\mathcal{O}$. Then we match the $K$-structure of these 
representations with $R(\mathcal{O}, \psi)$. This demonstrates the conjecture we state at the beginning of the introduction. The following theorem summarizes this.

Theorem 1.2. With notation as above, view $G_{0}=\operatorname{Spin}(2 n, \mathbb{C})$ as a real group. The $K$-structure of each representation in $\mathcal{U}_{G_{0}}\left(\mathcal{O}, \lambda_{\mathcal{O}}\right)$ is calculated explicitly and matches the $K$-structure of the $R(\mathcal{O}, \psi)$ with $\psi \in \widehat{A_{K}(\mathcal{O})}$. That is, there is a 1-1 correspondence $\psi \in \widehat{A_{K}(\mathcal{O})} \longleftrightarrow \Xi(\mathcal{O}, \psi) \in \mathcal{U}_{G_{0}}\left(\mathcal{O}, \lambda_{\mathcal{O}}\right)$ satisfying

$$
\left.\Xi(\mathcal{O}, \psi)\right|_{K} \cong R(\mathcal{O}, \psi) \text {. }
$$

For the case $O(2 n, \mathbb{C})$ (rather than $\operatorname{Spin}(2 n, \mathbb{C})$ ), the $K$-structure of the representations studied in this paper were considered earlier in [McG94 and BP11]. The unitarity of modules for the Spin groups (in particular the ones in this paper) is established in Bre99.

\section{Preliminaries}

2.1. Nilpotent orbits. The complex nilpotent orbits of type $D_{n}$ are parametrized by partitions of $2 n$, with even blocks occurring with even multiplicities, and with $I, I I$ in the very even case (see CM93]). The small nilpotent orbits satisfying (1.1.2) are those $\mathcal{O}$ with $\operatorname{dim} \mathcal{O} \leq n^{2}$.

We list them out as the following four cases:

\begin{tabular}{|c|c|c|}
\hline Case 1: $n=2 p$ & $\mathcal{O}=\left[\begin{array}{lll}3 & 2^{n-2} & 1\end{array}\right]$ & $\operatorname{dim} \mathcal{O}=n^{2}$ \\
\hline $\begin{aligned} \text { Case } 2: & n-2 p \\
& \text { or } 2 p+1\end{aligned}$ & 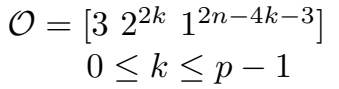 & $\begin{aligned} \operatorname{dim} \mathcal{O}= & 4 n k-4 k^{2} \\
& +4 n-8 k-4\end{aligned}$ \\
\hline Case 3: $n=2 p$ & $\mathcal{O}=\left[2^{n}\right]_{I, I I}$ & $\operatorname{dim} \mathcal{O}=n^{2}-n$ \\
\hline $\begin{aligned} \text { Case } 4: & n=2 p \\
& \text { or } 2 p+1\end{aligned}$ & $\begin{aligned} \mathcal{O}= & {\left[2^{2 k} 1^{2 n-4 k}\right] } \\
& 0 \leq k<n / 2\end{aligned}$ & $\operatorname{dim} \mathcal{O}=4 n k-4 k^{2}-2 k$ \\
\hline
\end{tabular}

Note that these are the orbits listed in [McG94. The proof of the next proposition, and the details about the nature of the component groups, are in Section 5 .

Proposition 2.2 (Corollary 5.4).

Case 1: If $\mathcal{O}=\left[\begin{array}{ll}3 & 2^{2 p-2} \\ 1\end{array}\right]$, then $A_{K}(\mathcal{O}) \cong \mathbb{Z}_{2} \times \mathbb{Z}_{2}$.

Case 2: If $\mathcal{O}=\left[\begin{array}{lll}3 & 2^{2 k} & 1^{2 n-4 k-3}\end{array}\right]$ with $2 n-4 k-3>1$, then $A_{K}(\mathcal{O}) \cong \mathbb{Z}_{2}$.

Case 3: If $\mathcal{O}=\left[2^{2 p}\right]_{I, I I}$, then $A_{K}(\mathcal{O}) \cong \mathbb{Z}_{2}$.

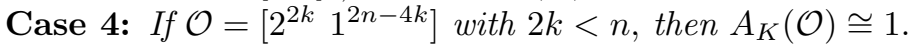

In all cases $C_{K}(\mathcal{O})=Z(K) \cdot C_{K}(\mathcal{O})^{0}$.

\section{Regular SECTiOnS}

We use the notation introduced in Sections 1 and 2. We compute the centralizers needed for $R(\mathcal{O}, \psi)$ in $\mathfrak{k}$ and in $K$. We use the standard roots and basis for $\mathfrak{s o}(2 n, \mathbb{C})$. A basis for the Cartan subalgebra is given by $H\left(\epsilon_{i}\right)$; the root vectors are $X\left( \pm \epsilon_{i} \pm\right.$ $\left.\epsilon_{j}\right)$. Realizations in terms of the Clifford algebra and explicit calculations are in Section 5 
Let $e$ be a representative of the orbit $\mathcal{O}$, and let $\{e, h, f\}$ be the corresponding Lie triple. Let

- $C_{\mathfrak{k}}(h)_{i}$ be the $i$-eigenspace of $\operatorname{ad}(h)$ in $\mathfrak{k}$,

- $C_{\mathfrak{k}}(e)_{i}$ be the $i$-eigenspace of $\operatorname{ad}(h)$ in the centralizer of $e$ in $\mathfrak{k}$,

- $C_{\mathfrak{k}}(h)^{+}:=\sum_{i>0} C_{\mathfrak{k}}(h)_{i}$ and $C_{\mathfrak{k}}(e)^{+}:=\sum_{i>0} C_{\mathfrak{k}}(e)_{i}$.

3.1. We describe the centralizer for $\mathcal{O}=\left[32^{2 k} 1^{2 n-4 k-3}\right]$ in detail. These are Cases 1 and 2. Representatives for $e$ and $h$ are

$$
\begin{aligned}
& e=X\left(\epsilon_{1}-\epsilon_{2 k+2}\right)+X\left(\epsilon_{1}+\epsilon_{2 k+2}\right)+\sum_{2 \leq i \leq 2 k+1} X\left(\epsilon_{i}+\epsilon_{k+i}\right), \\
& h=2 H\left(\epsilon_{1}\right)+\sum_{2 \leq i \leq 2 k+1} H\left(\epsilon_{i}\right)=H(2, \underbrace{1, \ldots, 1}_{2 k}, \underbrace{0, \ldots, 0}_{n-1-2 k}) .
\end{aligned}
$$

Then

$$
\begin{aligned}
& C_{\mathfrak{k}}(h)_{0}=\mathfrak{g l}(1) \times \mathfrak{g l}(2 k) \times \mathfrak{s o}(2 n-2-4 k), \\
& C_{\mathfrak{k}}(h)_{1}=\operatorname{Span}\left\{X\left(\epsilon_{1}-\epsilon_{i}\right), X\left(\epsilon_{i} \pm \epsilon_{j}\right), 2 \leq i \leq 2 k+1<j \leq n\right\}, \\
& C_{\mathfrak{k}}(h)_{2}=\operatorname{Span}\left\{X\left(\epsilon_{1} \pm \epsilon_{j}\right), X\left(\epsilon_{i}+\epsilon_{l}\right), 2 \leq i \neq l \leq 2 k+1<j \leq n\right\}, \\
& C_{\mathfrak{k}}(h)_{3}=\operatorname{Span}\left\{X\left(\epsilon_{1}+\epsilon_{i}\right), 2 \leq i \leq 2 k+1\right\} .
\end{aligned}
$$

Similarly

$$
\begin{aligned}
C_{\mathfrak{k}}(e)_{0} & \cong \mathfrak{s p}(2 k) \times \mathfrak{s o}(2 n-3-4 k), \\
C_{\mathfrak{k}}(e)_{1} & =\operatorname{Span}\left\{X\left(\epsilon_{1}-\epsilon_{i}\right)-X\left(\epsilon_{k+i} \pm \epsilon_{2 k+2}\right), X\left(\epsilon_{1}-\epsilon_{k+i}\right)-X\left(\epsilon_{i} \pm \epsilon_{2 k+2}\right),\right. \\
& \left.2 \leq i \leq k+1, X\left(\epsilon_{j} \pm \epsilon_{l}\right), 2 \leq j \leq 2 k+1,2 k+3 \leq l \leq n\right\}, \\
C_{\mathfrak{k}}(e)_{2} & =C_{\mathfrak{k}}(h)_{2}, \\
C_{\mathfrak{k}}(e)_{3} & =C_{\mathfrak{k}}(h)_{3} .
\end{aligned}
$$

We denote by $\chi$ the trivial character of $C_{\mathfrak{k}}(e)$. A representation of $K$ will be denoted by its highest weight:

$$
V=V\left(a_{1}, \ldots, a_{n}\right), \quad a_{1} \geq \cdots \geq\left|a_{n}\right|,
$$

with all $a_{i} \in \mathbb{Z}$ or all $a_{i} \in \mathbb{Z}+1 / 2$.

We will compute

(3.1.3) $\operatorname{Hom}_{C_{\mathfrak{k}}(e)}\left[V^{*}, \chi\right]=\operatorname{Hom}_{C_{\mathfrak{k}}(e)_{0}}\left[V^{*} /\left(C_{\mathfrak{k}}(e)^{+} V^{*}\right), \chi\right]:=\left[V^{*} /\left(C_{\mathfrak{k}}(e)^{+} V^{*}\right)\right]^{\chi}$.

3.2. Case 1. $n=2 p, \mathcal{O}=\left[\begin{array}{ll}3 & 2^{n-2} \\ 1\end{array}\right]$.

In this case $C_{\mathfrak{k}}(h)_{0}=\mathfrak{g l}(1) \times \mathfrak{g l}(n-2) \times \mathfrak{s o}(2), C_{\mathfrak{k}}(e)_{0}=\mathfrak{s p}(n-2)$.

Consider the parabolic $\mathfrak{p}=\mathfrak{l}+\mathfrak{n}$ determined by $h$,

$$
\begin{aligned}
\mathfrak{l} & =C_{\mathfrak{k}}(h)_{0} \cong \mathfrak{g l}(1) \times \mathfrak{g l}(n-2) \times \mathfrak{s o}(2), \\
\mathfrak{n} & =C_{\mathfrak{k}}(h)^{+} .
\end{aligned}
$$

We denote by $V^{*}$ the dual of $V$. Since $n=2 p, V^{*} \cong V$. If $V^{*}$ is a representation such that $\operatorname{Hom}_{C_{\mathfrak{k}}(e)}\left[V^{*}, \chi\right]$ in (3.1.3) is nonzero, then $V^{*}$ is a quotient of a generalized Verma module $M(\lambda)=U(\mathfrak{k}) \otimes_{U(\overline{\mathfrak{p}})} F(\lambda)$, where $\lambda$ is a weight of $V^{*}$ which is dominant for $\overline{\mathfrak{p}}$. This is

$$
\lambda=\left(-a_{1} ;-a_{n-1}, \ldots,-a_{2} ;-a_{n}\right) .
$$

The ; denotes the fact that this is a (highest) weight of $\mathfrak{l} \cong \mathfrak{g l}(1) \times \mathfrak{g l}(n-2) \times \mathfrak{s o}(2)$. 
We choose the standard positive root system $\triangle^{+}(\mathfrak{l})$ for $\mathfrak{l}$. As a $C_{\mathfrak{k}}(e)_{0}$-module,

$$
\mathfrak{n}=C_{\mathfrak{k}}(e)^{+} \oplus \mathfrak{n}^{\perp}
$$

where we can choose $\mathfrak{n}^{\perp}=\operatorname{Span}\left\{X\left(\epsilon_{1}-\epsilon_{j}\right), 2 \leq j \leq n-1\right\}$. This complement is l-invariant. It restricts to the standard module of $C_{\mathfrak{k}}(e)_{0}=\mathfrak{s} \mathfrak{p}(n-2)$.

The generalized Bernstein-Gelfand-Gelfand resolution is

$$
0 \cdots \longrightarrow \bigoplus_{w \in W^{+}, \ell(w)=k} M(w \cdot \lambda) \longrightarrow \cdots \longrightarrow \bigoplus_{w \in W^{+}, \ell(w)=1} M(w \cdot \lambda) \longrightarrow M(\lambda) \longrightarrow V^{*} \longrightarrow 0
$$

with $w \cdot \lambda:=w(\lambda+\rho(\mathfrak{k}))-\rho(\mathfrak{k})$, and $w \in W^{+}$, the $W(\mathfrak{l})$-coset representatives that make $w \cdot \lambda$ dominant for $\Delta^{+}(\mathfrak{l})$. This is a free $C_{\mathfrak{k}}(e)^{+}$-resolution so we can compute cohomology by considering

$$
0 \cdots \longrightarrow \bigoplus_{w \in W^{+}, \ell(w)=k} \overline{M(w \cdot \lambda)} \longrightarrow \cdots \longrightarrow \bigoplus_{w \in W^{+}, \ell(w)=1} \overline{M(w \cdot \lambda)} \longrightarrow \overline{M(\lambda)} \longrightarrow \overline{V^{*}} \longrightarrow 0
$$

where $\bar{X}$ denotes $X /\left[C_{\mathfrak{k}}(e)^{+} X\right]$.

Note that in the sequences, $M(w \cdot \lambda) \cong S(\mathfrak{n}) \otimes_{\mathbb{C}} F(w \cdot \lambda)$ and $\overline{M(w \cdot \lambda)} \cong$ $S\left(\mathfrak{n}^{\perp}\right) \otimes_{\mathbb{C}} F(w \cdot \lambda)$. As an $\mathfrak{l}$-module, $\mathfrak{n}^{\perp}$ has highest weight $(1 ; 0, \ldots, 0,-1 ; 0)$. Then $S^{k}\left(\mathfrak{n}^{\perp}\right) \cong F(k ; 0, \ldots, 0,-k ; 0)$ as an $\mathfrak{l}$-module.

Let $\mu:=\left(-\alpha_{1} ;-\alpha_{n-1}, \ldots,-\alpha_{2} ;-\alpha_{n}\right)$ be the highest weight of an l-module. By the Pieri's rule,

$$
S^{k}\left(\mathfrak{n}^{\perp}\right) \otimes F_{\mu}=\sum V\left(-\alpha_{1}+k ;-\alpha_{n-1}-k_{n-1}, \ldots,-\alpha_{3}-k_{3},-\alpha_{2}-k_{2} ;-\alpha_{n}\right) .
$$

The sum is taken over

$$
\left\{k_{i} \mid k_{i} \geq 0, \sum k_{i}=k, 0 \leq k_{i} \leq \alpha_{i-1}-\alpha_{i}, 3 \leq i \leq n-1\right\} .
$$

Lemma 3.3. $\operatorname{Hom}_{C_{\mathfrak{k}}(e)_{0}}\left[S\left(\mathfrak{n}^{\perp}\right) \otimes F_{\mu}: \chi\right] \neq 0$ for every $\mu$. The multiplicity is 1 .

Proof. Since $(\mathfrak{g l}(n-2), \mathfrak{s p}(n-2))$ is a hermitian symmetric pair, the theorem of Cartan and Helgason (cf. Theorem 3.3.1.1 in War72]) implies that a composition factor in the terms of $S\left(\mathfrak{n}^{\perp}\right) \otimes F_{\mu}$ in (3.2.4) admits $C_{\mathfrak{k}}(e)_{0}$-fixed vectors only if

$-\alpha_{n-1}-k_{n-1}=-\alpha_{n-2}-k_{n-2},-\alpha_{n-3}-k_{n-3}=-\alpha_{n-4}-k_{n-4}, \ldots,-\alpha_{3}-k_{3}=-\alpha_{2}-k_{2}$.

The conditions $0 \leq k_{i} \leq \alpha_{i-1}-\alpha_{i}$ imply

$$
k_{n-2}=0, \quad k_{n-1}=\alpha_{n-2}-\alpha_{n-1},
$$

$$
\begin{aligned}
& k_{4}=0, \quad k_{5}=\alpha_{4}-\alpha_{5}, \\
& k_{2}=0, \quad k_{3}=\alpha_{2}-\alpha_{3} .
\end{aligned}
$$

Therefore, given $\mu$, the weight of the $C_{\mathfrak{k}}(e)_{0}$-fixed vector in $S\left(\mathfrak{n}^{\perp}\right) \otimes F_{\mu}$ is $\left(-\alpha_{1}+\alpha_{2}-\alpha_{3}+\alpha_{4}-\alpha_{5}+\cdots+\alpha_{n-2}-\alpha_{n-1} ;-\alpha_{n-2},-\alpha_{n-2}, \ldots,-\alpha_{2}, \alpha_{2} ;-\alpha_{n}\right)$, and the multiplicity is 1 .

Corollary 3.4. For every $V\left(a_{1}, \ldots, a_{n}\right) \in \widehat{K}, \operatorname{Hom}_{C_{\mathfrak{k}}(e)}[V, \chi]=0$ or 1 . The action of ad $h$ is $-2 \sum_{1 \leq i \leq p} a_{2 i-1}$. 
Proof. The first statement follows from Lemma 3.3 and the surjection

$$
\overline{M(\lambda)} \cong S\left(\mathfrak{n}^{\perp}\right) \otimes_{\mathbb{C}} F(\lambda) \longrightarrow \overline{V^{*}} \longrightarrow 0 .
$$

The action of ad $h$ is computed from the module

$$
V\left(-a_{1}+k ;-a_{n-2},-a_{n-2}, \ldots,-a_{2},-a_{2} ;-a_{n}\right)
$$

with $k=a_{2}-a_{3}+a_{4}-a_{5}+\cdots+a_{n-2}-a_{n-1}$. The value is $-2 \sum_{1 \leq i \leq p} a_{2 i-1}$.

$\ell(\mathbf{w})=\mathbf{1}$. To show that the weights in (3.4.1) actually occur, it is enough to show that these weights do not occur in the term in the BGG resolution (3.2.3) with $\ell(w)=1$.

We calculate $w \cdot \lambda$ :

$$
\rho=\rho(\mathfrak{k})=(-(n-1) ;-1,-2, \ldots,-(n-2) ; 0)
$$

is dominant for $\overline{\mathfrak{p}}$, and

$$
\lambda+\rho=\left(-a_{1}-n+1 ;-a_{n-1}-1,-a_{n-2}-2, \ldots,-a_{2}-n+2 ;-a_{n}\right) .
$$

There are three elements $w \in W^{+}$of length 1 . They are the left $W(\mathfrak{l})$-cosets of

$$
w_{1}=s_{\epsilon_{1}-\epsilon_{n-1}}, w_{2}=s_{\epsilon_{2}-\epsilon_{n}}, w_{3}=s_{\epsilon_{2}+\epsilon_{n}} .
$$

So

$$
\begin{aligned}
& w_{1} \cdot \lambda=\left(-a_{2}+1 ;-a_{n-1},-a_{n-2}, \ldots,-a_{4},-a_{3},-a_{1}-1 ;-a_{n}\right), \\
& w_{2} \cdot \lambda=\left(-a_{1} ;-a_{n}+1,-a_{n-2},-a_{n-3}, \ldots,-a_{3},-a_{2} ;-a_{n-1}-1\right), \\
& w_{3} \cdot \lambda=\left(-a_{1} ; a_{n}+1,-a_{n-2},-a_{n-3}, \ldots,-a_{3},-a_{2} ; a_{n-1}+1\right) .
\end{aligned}
$$

Lemma 3.5. For all $\lambda, \operatorname{Hom}_{C_{\mathfrak{k}}(e)}\left[\overline{M\left(w_{i} \cdot \lambda\right)}, \chi\right]=1$. The eigenvalues of ad $h$ are different from $-2 \sum_{1 \leq i \leq p} a_{2 i-1}$ for each $w_{i}$.

Proof. The $\mathfrak{s p}(n-2)$-fixed weights coming from $S\left(\mathfrak{n}^{\perp}\right) \otimes F\left(w_{i} \cdot \lambda\right), i=1,2,3$, are

$$
\begin{gathered}
w_{1} \longleftrightarrow\left(a_{1}-a_{2}-a_{3}+a_{4}-a_{5}+\cdots+a_{n-2}-a_{n-1}+2 ;\right. \\
\left.-a_{n-2},-a_{n-2}, \ldots,-a_{4},-a_{4},-a_{1}-1,-a_{1}-1 ;-a_{n}\right) \\
\begin{array}{c}
w_{2} \longleftrightarrow\left(-a_{1}+a_{2}-a_{3}+\cdots+a_{n-4}-a_{n-3}+a_{n-2}-a_{n}+1 ;\right. \\
\left.-a_{n-2},-a_{n-2}, \ldots,-a_{4},-a_{4},-a_{2},-a_{2} ;-a_{n-1}-1\right) \\
w_{3} \longleftrightarrow\left(-a_{1}+a_{2}-a_{3}+\cdots+a_{n-4}-a_{n-3}+a_{n-2}+a_{n}+1 ;\right. \\
\left.-a_{n-2},-a_{n-2}, \ldots,-a_{4},-a_{4},-a_{2},-a_{2} ; a_{n-1}-1\right)
\end{array}
\end{gathered}
$$

The negatives of the weights of $h$ are

$$
\begin{aligned}
& w_{0}=1 \longleftrightarrow 2\left(a_{1}+a_{3}+\cdots+a_{n-1}\right), \\
& w_{1} \longleftrightarrow 2\left(a_{2}+a_{3}+a_{5} \cdots+a_{n-1}-1\right), \\
& w_{2} \longleftrightarrow 2\left(a_{1}+a_{3}+\cdots+a_{n-3}+a_{n}-1\right), \\
& w_{3} \longleftrightarrow 2\left(a_{1}+a_{3}+\cdots+a_{n-3}-a_{n}-1\right) .
\end{aligned}
$$

The last three weights are not equal to the first one. This completes the proof. 
Theorem 3.6. Let $K=\operatorname{Spin}(2 n, \mathbb{C})$ with $n=2 p$. Every representation $V\left(a_{1}, \ldots, a_{n}\right)$ has $C_{\mathfrak{k}}(e)$ fixed vectors and the multiplicity is 1. We write $C_{K}(\mathcal{O}):=C_{K}(e)$. In summary,

$$
\operatorname{Ind}_{C_{K}(\mathcal{O})^{0}}^{K}(\text { Triv })=\bigoplus_{a \in \widehat{K}} V\left(a_{1}, \ldots, a_{n}\right) .
$$

Theorem 3.6 can be interpreted as computing regular functions on the universal cover $\widetilde{\mathcal{O}}$ of $\mathcal{O}$ transforming trivially under $C_{\mathfrak{k}}(e)_{0}$. We decompose it further:

$$
R(\widetilde{\mathcal{O}}, \operatorname{Triv}):=\operatorname{Ind}_{C_{K}(\mathcal{O})^{0}}^{K}(\text { Triv })=\operatorname{Ind}_{C_{K}(\mathcal{O})}^{K}\left[\operatorname{Ind}_{C_{K}(\mathcal{O})^{0}}^{C_{K}(\mathcal{O})}(\text { Triv })\right] .
$$

The inner induced module splits into

$$
\operatorname{Ind}_{C_{K}(\mathcal{O})^{0}}^{C_{K}(\mathcal{O})}(\text { Triv })=\sum \psi
$$

where $\psi$ are the irreducible representations of $C_{K}(\mathcal{O})$ trivial on $C_{K}(\mathcal{O})^{0}$. Thus, the sum in $(3.6 .2)$ is taken over $\widehat{A_{K}(\mathcal{O})}$.

Then

$$
R(\widetilde{\mathcal{O}}, \text { Triv })=\operatorname{Ind}_{C_{K}(\mathcal{O})^{0}}^{K}(\text { Triv })=\sum_{\psi \in \widehat{A_{K}(\mathcal{O})}} R(\mathcal{O}, \psi)
$$

We will decompose $R(\mathcal{O}, \psi)$ explicitly as a representation of $K$.

Lemma 3.7. Let $K=\operatorname{Spin}(2 n, \mathbb{C})$ with $n=2 p$. Let $\mu_{i}, 1 \leq i \leq 4$, be the following $K$-types parametrized by their highest weights:

$$
\begin{gathered}
\mu_{1}=(0, \ldots, 0), \mu_{2}=(1,0, \ldots, 0), \\
\mu_{3}=\left(\frac{1}{2}, \ldots, \frac{1}{2}\right), \mu_{4}=\left(\frac{1}{2}, \ldots, \frac{1}{2},-\frac{1}{2}\right) .
\end{gathered}
$$

Let $\psi_{i}$ be the restriction of the highest weight of $\mu_{i}$ to $C_{K}(\mathcal{O})$, respectively. Then

$$
\operatorname{Ind}_{C_{K}(\mathcal{O})^{0}}^{C_{K}(\mathcal{O})}(\text { Triv })=\sum_{i=1}^{4} \psi_{i} .
$$

Proposition 3.8. Let $K=\operatorname{Spin}(2 n, \mathbb{C})$ with $n=2 p$. The induced representation (3.6.3) decomposes as

$$
\operatorname{Ind}_{C_{K}(\mathcal{O})}^{K}(\text { Triv })=\sum_{i=1}^{4} R\left(\mathcal{O}, \psi_{i}\right)
$$

where

$$
\begin{aligned}
& R\left(\mathcal{O}, \psi_{1}\right)=\operatorname{Ind}_{C_{K}(\mathcal{O})}^{K}\left(\psi_{1}\right)=\bigoplus V\left(a_{1}, \ldots, a_{n}\right) \text { with } a_{i} \in \mathbb{Z}, \sum a_{i} \in 2 \mathbb{Z}, \\
& R\left(\mathcal{O}, \psi_{2}\right)=\operatorname{Ind}_{C_{K}(\mathcal{O})}^{K}\left(\psi_{2}\right)=\bigoplus V\left(a_{1}, \ldots, a_{n}\right) \text { with } a_{i} \in \mathbb{Z}, \sum a_{i} \in 2 \mathbb{Z}+1, \\
& R\left(\mathcal{O}, \psi_{3}\right)=\operatorname{Ind}_{C_{K}(\mathcal{O})}^{K}\left(\psi_{3}\right)=\bigoplus V\left(a_{1}, \ldots, a_{n}\right) \text { with } a_{i} \in \mathbb{Z}+1 / 2, \sum a_{i} \in 2 \mathbb{Z}+p, \\
& R\left(\mathcal{O}, \psi_{4}\right)=\operatorname{Ind}_{C_{K}(\mathcal{O})}^{K}\left(\psi_{4}\right)=\bigoplus V\left(a_{1}, \ldots, a_{n}\right) \text { with } a_{i} \in \mathbb{Z}+1 / 2, \sum a_{i} \in 2 \mathbb{Z}+p+1 .
\end{aligned}
$$


3.9. Case 2: $\mathcal{O}=\left[\begin{array}{lll}3 & 2^{2 k} & 1^{2 n-4 k-3}\end{array}\right], 0 \leq k \leq p-1$.

Consider the parabolic $\mathfrak{p}=\mathfrak{l}+\mathfrak{n}$ determined by $h$ :

$$
\begin{aligned}
\mathfrak{l} & =C_{\mathfrak{k}}(h)_{0} \cong \mathfrak{g l}(1) \times \mathfrak{g l}(2 k) \times \mathfrak{s o}(2 n-2-4 k), \\
\mathfrak{n} & =C_{\mathfrak{k}}(h)^{+} .
\end{aligned}
$$

In this section, let $\epsilon=-1$ when $n$ is even; $\epsilon=1$ when $n$ is odd. The dual of $V$, denoted $V^{*}$, has lowest weight $\left(\epsilon a_{n},-a_{n-1}, \ldots,-a_{2},-a_{1}\right)$. It is therefore a quotient of a generalized Verma module $M(\lambda)=U(\mathfrak{k}) \otimes_{U(\overline{\mathfrak{p}})} F(\lambda)$, where $\lambda$ is dominant for $\overline{\mathfrak{p}}$ and dominant for the standard positive system for $\mathfrak{l}$ :

$$
\lambda=(-a_{1} ; \underbrace{-a_{2 k+1}, \ldots,-a_{3},-a_{2}}_{2 k} ; \underbrace{a_{2 k+2}, \ldots, a_{n-1}, \epsilon a_{n}}_{n-1-2 k}) .
$$

$\mathfrak{n}=C_{\mathfrak{k}}(e)^{+} \oplus \mathfrak{n}^{\perp}$ as a module for $C_{\mathfrak{k}}(e)_{0}$. A basis for $\mathfrak{n}^{\perp} \subset C_{\mathfrak{k}}(h)_{1}$ is given by

$$
\left\{X\left(\epsilon_{1}-\epsilon_{2 k+2}\right)\right\}, \quad 2 \leq i \leq 2 k+1 .
$$

This is the standard representation of $\mathfrak{s p}(2 k)$, trivial for $\mathfrak{s o}(2 n-4-4 k)$. We write its highest weight as

$$
(1 ; 0, \ldots, 0,-1 ; 0, \ldots, 0) .
$$

We can now repeat the argument for the case $k=p$; there is an added constraint that $a_{2 k+3}=\cdots=a_{n}=0$ because the representation with highest weight $\left(a_{2 k+2}, \ldots, a_{n-1}, \epsilon a_{n}\right)$ of $\mathfrak{s o}(2 n-2-4 k)$ must have fixed vectors for $\mathfrak{s o}(2 n-3-4 k)$.

Then the next theorem follows.

Theorem 3.10. A representation $V\left(a_{1}, \ldots, a_{n}\right)$ has $C_{\mathfrak{k}}(e)$ fixed vectors if and only if

$$
a_{2 k+3}=\cdots=a_{n}=0,
$$

and the multiplicity is 1 . In summary,

$\operatorname{Ind}_{C_{K}(\mathcal{O})^{0}}^{K}($ Triv $)=\bigoplus V\left(a_{1}, \ldots, a_{2 k+2}, 0 \ldots, 0\right)$, with $a_{1} \geq \cdots \geq a_{2 k+2} \geq 0, a_{i} \in \mathbb{Z}$.

As in (3.6.3), we decompose $\operatorname{Ind}_{C_{K}(\mathcal{O})^{0}}^{K}($ Triv) further into the sum of $R(\mathcal{O}, \psi)$ with $\psi \in \widehat{A_{K}(\mathcal{O})}$.

Lemma 3.11. Let $\mu_{1}, \mu_{2}$ be the following $K$-types parametrized by their highest weights:

$$
\mu_{1}=(0, \ldots, 0), \mu_{2}=(1,0, \ldots, 0) .
$$

Let $\psi_{i}$ be the restriction of the highest weight of $\mu_{i}$ to $C_{G}(\mathcal{O})$, respectively. Then

$$
\operatorname{Ind}_{C_{K}(\mathcal{O})^{0}}^{C_{K}(\mathcal{O})}(\text { Triv })=\psi_{1}+\psi_{2} .
$$

Proposition 3.12. The induced representation (3.6.3) decomposes as

$$
\operatorname{Ind}_{C_{K}(\mathcal{O})^{0}}^{K}(\text { Triv })=R\left(\mathcal{O}, \psi_{1}\right)+R\left(\mathcal{O}, \psi_{2}\right) \text {, }
$$

where

$R\left(\mathcal{O}, \psi_{1}\right)=\operatorname{Ind}_{C_{K}(\mathcal{O})}^{K}\left(\psi_{1}\right)=\bigoplus V\left(a_{1}, \ldots, a_{2 k+2}, 0, \ldots, 0\right)$ with $a_{i} \in \mathbb{Z}, \sum a_{i} \in 2 \mathbb{Z}$,
$R\left(\mathcal{O}, \psi_{2}\right)=\operatorname{Ind}_{C_{K}(\mathcal{O})}^{K}\left(\psi_{2}\right)=\bigoplus V\left(a_{1}, \ldots, a_{2 k+2}, 0, \ldots, 0\right)$ with $a_{i} \in \mathbb{Z}, \sum a_{i} \in 2 \mathbb{Z}+1$. 
3.13. Now we treat $\mathcal{O}=\left[2^{2 k} 1^{2 n-4 k}\right]$ with $0 \leq k \leq p$. These are Cases 3 and 4 . When $k=p$ (and hence $n=2 p$ ), the orbit is labeled by $I, I I$. The computation is similar and easier than the previous two cases. We state the results for $R(\widetilde{O}$, Triv $)$ as follows.

\section{Theorem 3.14.}

Case 3: For $k=p$, so $n=2 p$,

$$
\begin{aligned}
\mathcal{O}_{I}=\left[2^{n}\right]_{I}, & R\left(\widetilde{\mathcal{O}_{I}}, \text { Triv }\right) \\
& =\operatorname{Ind}_{C_{K}\left(\mathcal{O}_{I}\right)^{0}}^{K}(\text { Triv }) \\
& =\bigoplus V\left(a_{1}, a_{1}, a_{3}, a_{3}, \ldots, a_{n-1}, a_{n-1}\right), \\
\mathcal{O}_{I I}=\left[2^{n}\right]_{I I}, \quad R\left(\widetilde{\mathcal{O}_{I I}}, \text { Triv }\right) & =\operatorname{Ind}_{C_{K}\left(\mathcal{O}_{I I}\right)^{0}}^{K}(\text { Triv }) \\
& =\bigoplus V\left(a_{1}, a_{1}, a_{3}, a_{3}, \ldots, a_{n-1},-a_{n-1}\right) .
\end{aligned}
$$

Case 4: For $k \leq p-1$,

$$
\begin{aligned}
& \mathcal{O}=\left[2^{2 k} 1^{2 n-4 k}\right], \quad R(\widetilde{\mathcal{O}}, \text { Triv })=\operatorname{Ind}_{C_{K}(\mathcal{O})^{0}}^{K}(\text { Triv }) \\
& =\bigoplus V\left(a_{1}, a_{1}, a_{3}, a_{3}, \ldots, a_{2 k-1}, a_{2 k-1}, 0, \ldots, 0\right), \\
& \text { satisfying } a_{1} \geq a_{3} \geq \cdots \geq a_{2 k-1} \geq 0 \text {. }
\end{aligned}
$$

Proof. We treat the case $n=2 p$ and $k \leq p-1 ; n=2 p+1$ is similar. A representative of $\mathcal{O}$ is $e=X\left(\epsilon_{1}+\epsilon_{2}\right)+\cdots+X\left(\epsilon_{2 k-1}+\epsilon_{2 k}\right)$, and the corresponding middle element in the Lie triple is $h=H(\underbrace{1, \ldots, 1}_{2 k}, \underbrace{0, \ldots, 0}_{n-2 k})$. Thus

$$
\begin{aligned}
& C_{\mathfrak{k}}(h)_{0}=\mathfrak{g l}(2 k) \times \mathfrak{s o}(2 n-4 k), \\
& C_{\mathfrak{k}}(h)_{1}=\operatorname{Span}\left\{X\left(\epsilon_{i} \pm \epsilon_{j}\right)\right\}, \quad 1 \leq i \leq 2 k<j \leq n, \\
& C_{\mathfrak{k}}(h)_{2}=\operatorname{Span}\left\{X\left(\epsilon_{l}+\epsilon_{m}\right)\right\}, \quad 1 \leq l \neq m \leq 2 k .
\end{aligned}
$$

and

$$
\begin{aligned}
C_{\mathfrak{k}}(e)_{0} & =\mathfrak{s p}(2 k) \times \mathfrak{s o}(2 n-4 k), \\
C_{\mathfrak{k}}(e)_{1} & =C_{\mathfrak{k}}(h)_{1}, \\
C_{\mathfrak{k}}(e)_{2} & =C_{\mathfrak{k}}(h)_{2} .
\end{aligned}
$$

As before, let $\mathfrak{p}=\mathfrak{l}+\mathfrak{n}$ be the parabolic subalgebra determined by $h$, and let $V=V\left(a_{1}, \ldots, a_{n}\right)$ be an irreducible representation of $K$. Since we assumed $n=2 p$, $V=V^{*}$. In this case $C_{\mathfrak{k}}(e)^{+}=\mathfrak{n}$, so Kostant's theorem implies $V /\left[C_{\mathfrak{k}}(e)^{+} V\right]=$ $V_{\mathfrak{l}}\left(a_{1}, \ldots a_{2 k} ; a_{2 k+1}, \ldots, a_{n}\right)$ as a $\mathfrak{g l}(2 k) \times \mathfrak{s o}(2 n-4 k)$-module. Since we want $\mathfrak{s p}(2 k) \times \mathfrak{s o}(2 n-4 k)$-fixed vectors, $a_{2 k+1}=\cdots=a_{n}=0$, and Cartan and Helgason's theorem implies $a_{1}=a_{2}, a_{3}=a_{4}, \ldots, a_{2 k-1}=a_{2 k}$.

When $n=2 p$ and $\mathcal{O}=\left[2^{n}\right]_{I, I I}$, the calculations are similar to $k \leq p-1$. The choices $I, I I$ are

$$
\begin{gathered}
e_{I}=X\left(\epsilon_{1}-\epsilon_{2}\right)+X\left(\epsilon_{3}-\epsilon_{4}\right)+\cdots+X\left(\epsilon_{n-1}-\epsilon_{n}\right), \\
h_{I}=H(1, \ldots, 1), \\
e_{I I}=X\left(\epsilon_{1}-\epsilon_{2}\right)+X\left(\epsilon_{3}-\epsilon_{4}\right)+\cdots+X\left(\epsilon_{n-3}-\epsilon_{n-2}\right)+X\left(\epsilon_{n-1}+\epsilon_{n}\right), \\
h_{I I}=H(1, \ldots, 1,-1) .
\end{gathered}
$$


These orbits are induced from the two nonconjugate maximal parabolic subalgebras with $\mathfrak{g l}(n)$ as Levi components, and $R\left(\widetilde{\mathcal{O}_{I, I I}}\right.$, Triv $)$ are just the induced modules from the trivial representation on the Levi component.

We aim at decomposing $R(\widetilde{\mathcal{O}}$, Triv $)=\sum R(\mathcal{O}, \psi)$ with $\psi \in \widehat{A_{K}(\mathcal{O})}$ as before.

\section{Lemma 3.15.}

Case 3: $n=2 p, \mathcal{O}=\left[2^{n}\right]_{I, I I}$. Let $\mu_{1}, \mu_{2}, \nu_{1}, \nu_{2}$, be

$$
\begin{gathered}
\mu_{1}=(1, \ldots, 1), \mu_{2}=\left(\frac{1}{2}, \ldots \frac{1}{2}\right), \\
\nu_{1}=(1, \ldots, 1,-1), \nu_{2}=\left(\frac{1}{2}, \ldots, \frac{1}{2},-\frac{1}{2}\right) .
\end{gathered}
$$

Let $\psi_{i}$ be the restriction of the highest weight of $\mu_{i}$ to $C_{K}(e)$, and let $\phi_{i}$ be the restriction of the highest weight of $\nu_{i}$, respectively. Then

$$
\begin{aligned}
\operatorname{Ind}_{C_{K}\left(\mathcal{O}_{I}\right)^{0}}^{C_{K}\left(\mathcal{O}_{I}\right)}(\text { Triv }) & =\psi_{1}+\psi_{2}, \\
\operatorname{Ind}_{C_{K}\left(\mathcal{O}_{I I}\right)^{0}}^{C_{K}\left(\mathcal{O}_{I}\right)}(\text { Triv }) & =\phi_{1}+\phi_{2} .
\end{aligned}
$$

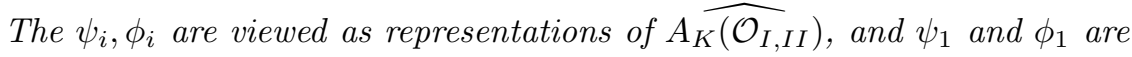
Triv, and $\psi_{2}, \phi_{2}$ are Sgn.

Case $4: \mathcal{O}=\left[2^{2 k} 1^{2 n-4 k}\right], 0 \leq k \leq p-1$.

$$
\operatorname{Ind}_{C_{K}(\mathcal{O})^{0}}^{C_{K}(\mathcal{O})}(\text { Triv })=\text { Triv. }
$$

Then we are able to split up $R(\widetilde{\mathcal{O}}$, Triv) as a sum of $R(\mathcal{O}, \psi)$ as in (3.6.3).

\section{Proposition 3.16.}

Case 3: $n=2 p, \mathcal{O}=\left[2^{n}\right]_{I, I I}: R\left(\widetilde{\mathcal{O}}_{I, I I}\right)=R\left(\mathcal{O}_{I, I I}\right.$, Triv $)+R\left(\mathcal{O}_{I, I I}\right.$, Sgn $)$ with

$$
\begin{aligned}
R\left(\mathcal{O}_{I}, \text { Triv }\right) & =\operatorname{Ind}_{C_{K}\left(\mathcal{O}_{I}\right)}^{K}(\text { Triv }) \\
& =\bigoplus V\left(a_{1}, a_{1}, a_{3}, a_{3}, \ldots, a_{n-1}, a_{n-1}\right), \quad \text { with } a_{i} \in \mathbb{Z}, \\
R\left(\mathcal{O}_{I}, \text { Sgn }\right) & =\operatorname{Ind}_{C_{K}\left(\mathcal{O}_{I}\right)}^{K}(\text { Sgn }) \\
& =\bigoplus V\left(a_{1}, a_{1}, a_{3}, a_{3}, \ldots, a_{n-1}, a_{n-1}\right), \quad \text { with } a_{i} \in \mathbb{Z}+1 / 2, \\
R\left(\mathcal{O}_{I I}, \text { Triv }\right) & =\operatorname{Ind}_{C_{K}\left(\mathcal{O}_{I I}\right)}^{K}(\text { Triv }) \\
& =\bigoplus V\left(a_{1}, a_{1}, a_{3}, a_{3}, \ldots, a_{n-1},-a_{n-1}\right), \quad \text { with } a_{i} \in \mathbb{Z}, \\
R\left(\mathcal{O}_{I I}, S g n\right) & =\operatorname{Ind}_{C_{K}\left(\mathcal{O}_{I I}\right)}^{K}(\text { Sgn }) \\
& =\bigoplus V\left(a_{1}, a_{1}, a_{3}, a_{3}, \ldots, a_{n-1},-a_{n-1}\right), \quad \text { with } a_{i} \in \mathbb{Z}+1 / 2,
\end{aligned}
$$

satisfying $a_{1} \geq a_{3} \geq \cdots \geq a_{n-1} \geq 0$.

Case 4: $\mathcal{O}=\left[2^{2 k} 1^{2 n-4 k}\right], 0 \leq k \leq p-1:$

$$
\begin{aligned}
R(\widetilde{\mathcal{O}}, \text { Triv }) & =R(\mathcal{O}, \text { Triv })=\operatorname{Ind}_{C_{K}(\mathcal{O})}^{K}(\text { Triv }) \\
& =\bigoplus V\left(a_{1}, a_{1}, a_{3}, a_{3}, \ldots, a_{2 k-1}, a_{2 k-1}, 0, \ldots, 0\right), \text { with } a_{i} \in \mathbb{Z},
\end{aligned}
$$

satisfying $a_{1} \geq a_{3} \geq \cdots \geq a_{2 k-1} \geq 0$. 


\section{Representations With SMALl SUpporT}

4.1. Langlands classification. Let $G$ be a complex linear algebraic reductive group viewed as a real Lie group. Let $\theta$ be a Cartan involution with fixed points $K$. Let $G \supset B=H N \supset H=T A$ be a Borel subgroup containing a fixed $\theta$-stable Cartan subalgebra $H$, with

$$
\begin{aligned}
& T=\{h \in H \quad \mid \quad \theta(h)=h\}, \\
& A=\left\{h \in H \quad \mid \quad \theta(h)=h^{-1}\right\} .
\end{aligned}
$$

The Langlands classification is as follows. Let $\chi \in \widehat{H}$. Denote by

$$
X(\chi):=\operatorname{Ind}_{B}^{G}[\chi \otimes \mathbb{1}]_{K \text {-finite }}
$$

the corresponding admissible standard module (Harish-Chandra induction). Let $(\mu, \nu)$ be the differentials of $\left.\chi\right|_{T}$ and $\left.\chi\right|_{A}$, respectively. Let $\lambda_{L}=(\mu+\nu) / 2$, and let $\lambda_{R}=(\mu-\nu) / 2$. We write $X(\mu, \nu)=X\left(\lambda_{L}, \lambda_{R}\right)=X(\chi)$.

\section{Theorem 4.2.}

(1) $X(\mu, \nu)$ has a unique irreducible subquotient denoted $\bar{X}(\mu, \nu)$ which contains the $K$-type with extremal weight $\mu$ occurring with multiplicity one in $X(\mu, \nu)$.

(2) $\bar{X}(\mu, \nu)$ is the unique irreducible quotient when $\langle$ Re $\nu, \alpha\rangle>0$ for all $\alpha \in$ $\Delta(\mathfrak{n}, \mathfrak{h})$, and the unique irreducible submodule when $\langle$ Rev, $\alpha\rangle<0$.

(3) $\bar{X}(\mu, \nu) \cong \bar{X}\left(\mu^{\prime}, \nu^{\prime}\right)$ if and only if there is $w \in W$ such that $w \mu=\mu^{\prime}, w \nu=$ $\nu^{\prime}$. Similarly for $\left(\lambda_{L}, \lambda_{R}\right)$.

Assume $\lambda_{L}, \lambda_{R}$ are both dominant integral. Write $F(\lambda)$ to be the finitedimensional representation of $G$ with infinitesimal character $\lambda$. Then $\bar{X}\left(\lambda_{L},-\lambda_{R}\right)$ is the finite-dimensional representation $F\left(\lambda_{L}\right) \otimes F\left(-w_{0} \lambda_{R}\right)$ where $w_{0} \in W$ is the longest element in the Weyl group. The lowest $K$-type has extremal weight $\lambda_{L}-\lambda_{R}$. Weyl's character formula implies

$$
\bar{X}\left(\lambda_{L},-\lambda_{R}\right)=\sum_{w \in W} \epsilon(w) X\left(\lambda_{L},-w \lambda_{R}\right) .
$$

In the following contents in this section, we use different notation as follows. We write $(G, K)=(\operatorname{Spin}(2 n, \mathbb{C}), \operatorname{Spin}(2 n))$ and $(\underline{G}, \underline{K})=(\operatorname{SO}(2 n, \mathbb{C}), \operatorname{SO}(2 n))$.

4.3. Infinitesimal characters. From [Bar17, we can associate to each $\mathcal{O}$ in Section 2.1 an infinitesimal character $\lambda_{\mathcal{O}}$. The fact is that $\mathcal{O}$ is the minimal orbit which can be the associated variety of a $(\mathfrak{g}, K)$-module with infinitesimal character $\left(\lambda_{L}, \lambda_{R}\right)$, with $\lambda_{L}$ and $\lambda_{R}$ both conjugate to $\lambda_{\mathcal{O}}$. The $\lambda_{\mathcal{O}}$ are listed below.

Case 1: $n=2 p, \mathcal{O}=\left[\begin{array}{ll}32^{n-2} & 1\end{array}\right]$,

$$
\lambda_{\mathcal{O}}=\rho / 2=\left(p-\frac{1}{2}, \ldots, \frac{3}{2}, \frac{1}{2} \mid p-1, \ldots, 1,0\right) .
$$

Case 2: $\mathcal{O}=\left[\begin{array}{lll}3 & 2^{2 k} & 1^{2 n-4 k-3}\end{array}\right], 0 \leq k \leq p-1$,

$$
\lambda_{\mathcal{O}}=\left(k+\frac{1}{2}, \ldots, \frac{3}{2}, \frac{1}{2} \mid n-k-2, \ldots, 1,0\right) .
$$


Case 3: $n=2 p, \mathcal{O}_{I, I I}=\left[2^{n}\right]_{I, I I}$,

$$
\begin{aligned}
\lambda_{\mathcal{O}_{I}} & =\left(\frac{2 n-1}{4}, \frac{2 n-5}{4}, \ldots, \frac{-(2 n-7)}{4}, \frac{-(2 n-3)}{4}\right), \\
\lambda_{\mathcal{O}_{I I}} & =\left(\frac{2 n-1}{4}, \frac{2 n-5}{4}, \ldots, \frac{-(2 n-7)}{4}, \frac{(2 n-3)}{4}\right) .
\end{aligned}
$$

Case 4: $\mathcal{O}=\left[2^{2 k} 1^{2 n-4 k}\right], 0 \leq k \leq p-1$,

$$
\lambda_{\mathcal{O}}=(k, k-1, \ldots, 1 ; n-k-1, \ldots, 1,0) .
$$

Notice that the infinitesimal characters in Cases 1 and 2 are nonintegral. For instance, in Case $1, \lambda_{\mathcal{O}}=\rho / 2$, where $\rho$ is a half sum of the positive roots of type $D_{2 p}$. The integral system is of type $D_{p} \times D_{p}$. The notation | separates the coordinates of the two $D_{p}$.

4.4. We define the following irreducible modules in terms of Langlands classification:

Case 1: $n=2 p, \mathcal{O}=\left[\begin{array}{ll}32^{n-1} & 1]\end{array}\right.$.

(i) $\Xi_{1}=\bar{X}\left(\lambda_{\mathcal{O}},-\lambda_{\mathcal{O}}\right)$;

(ii) $\Xi_{2}=\bar{X}\left(\lambda_{\mathcal{O}},-w_{1} \lambda_{\mathcal{O}}\right)$, where $w_{1} \lambda_{\mathcal{O}}=\left(p-\frac{1}{2}, \ldots, \frac{3}{2},-\frac{1}{2} \mid p-1, \ldots, 1,0\right)$;

(iii) $\Xi_{3}=\bar{X}\left(\lambda_{\mathcal{O}},-w_{2} \lambda_{\mathcal{O}}\right)$, where $w_{2} \lambda_{\mathcal{O}}=\left(p-1, \ldots, 1,0 \mid p-\frac{1}{2}, \ldots, \frac{3}{2}, \frac{1}{2}\right)$;

(iv) $\Xi_{4}=\bar{X}\left(\lambda_{\mathcal{O}},-w_{3} \lambda_{\mathcal{O}}\right)$, where $w_{3} \lambda_{\mathcal{O}}=\left(p-1, \ldots, 1,0 \mid p-\frac{1}{2}, \ldots, \frac{3}{2},-\frac{1}{2}\right)$.

Case 2: $\mathcal{O}=\left[\begin{array}{lll}3 & 2^{2 k} & 1^{2 n-4 k-3}\end{array}\right], 0 \leq k \leq p-1$.

(i) $\Xi_{1}=\bar{X}\left(\lambda_{\mathcal{O}},-\lambda_{\mathcal{O}}\right)$;

(ii) $\Xi_{2}=\bar{X}\left(\lambda_{\mathcal{O}},-w_{1} \lambda_{\mathcal{O}}\right), w_{1} \lambda_{\mathcal{O}}=\left(k+\frac{1}{2}, \ldots, \frac{3}{2}, \frac{1}{2} \mid n-k-2, \ldots, 1,0\right)$.

Case 3: $n=2 p, \mathcal{O}_{I, I I}=\left[2^{n}\right]_{I, I I}$.

(i) $\Xi_{I}=\bar{X}\left(\lambda_{\mathcal{O}_{I}},-\lambda_{\mathcal{O}_{I}}\right)$;

(i') $\Xi_{I}=\bar{X}\left(\lambda_{\mathcal{O}_{I}},-w \lambda_{\mathcal{O}_{I}}\right), w \lambda_{\mathcal{O}_{I}}=\left(\frac{2 n-3}{4}, \frac{2 n-7}{4}, \ldots, \frac{-(2 n-5)}{4}, \frac{-(2 n-1)}{4}\right)$;

(ii) $\Xi_{I I}=\bar{X}\left(\lambda_{\mathcal{O}_{I I}},-\lambda_{\mathcal{O}_{I I}}\right)$;

(ii') $\Xi_{I I}^{\prime}=\bar{X}\left(\lambda_{\mathcal{O}_{I I}},-w \lambda_{\mathcal{O}_{I I}}\right), w \lambda_{\mathcal{O}_{I I}}=\left(\frac{2 n-3}{4}, \frac{2 n-7}{4}, \ldots, \frac{-(2 n-5)}{4}, \frac{2 n-1}{4}\right)$.

Case 4: $\mathcal{O}=\left[2^{2 k} 1^{2 n-4 k}\right], 0 \leq k \leq p-1$.

(i) $\Xi=\bar{X}\left(\lambda_{\mathcal{O}},-\lambda_{\mathcal{O}}\right)$.

Remark 4.5. The representations introduced above form the set $\mathcal{U}_{G}\left(\mathcal{O}, \lambda_{\mathcal{O}}\right)$. The integral systems are of type $D_{p} \times D_{p}$ in Case $1, D_{k+1} \times D_{n-k-1}$ in Case $2, A_{n}$ in Case 3 , and $D_{n}$ in Case 4 . It is then a matter of computing the multiplicity of the sgn representation in the corresponding primitive ideal double cells for these integral systems. We omit the details.

Notation. We write $F(\lambda)$ for the finite-dimensional representation of the appropriate SO or Spin group with infinitesimal character $\lambda$. Write $V(\mu)$ for the finitedimensional representation of the appropriate SO or Spin group with highest weight $\mu$.

4.6. $K$-structure. We compute the $K$-types of each representation listed in Subsection 4.4 
Case 1: The arguments are refinements of those in [McG94. Let $H$ be the image of $\operatorname{Spin}(2 p, \mathbb{C}) \times \operatorname{Spin}(2 p, \mathbb{C})$ in $\operatorname{Spin}(4 p, \mathbb{C})$, and $U$ the image of the maximal compact subgroup $\operatorname{Spin}(2 p) \times \operatorname{Spin}(2 p)$ in $K$. Irreducible representations of $U$ can be viewed as $\operatorname{Spin}(2 p) \times \operatorname{Spin}(2 p)$-representations such that $\pm(I, I)$ acts trivially.

Cases (i) and (ii) factor to representations of $\mathrm{SO}(2 n, \mathbb{C})$, and (iii) and (iv) are genuine for $\operatorname{Spin}(2 n, \mathbb{C})$.

The Kazhdan-Lusztig conjectures for a nonintegral infinitesimal character (cf. ABV92, Chapters 16 and 17]) together with Weyl's formula for the character of a finite-dimensional module (see (4.2.1)) imply that

$$
\bar{X}\left(\rho / 2,-w_{i} \rho / 2\right)=\sum_{w \in W\left(D_{p} \times D_{p}\right)} \epsilon(w) X\left(\rho / 2,-w w_{i} \rho / 2\right),
$$

since $W\left(\lambda_{\mathcal{O}}\right)=W\left(D_{p} \times D_{p}\right)$.

Restricting (4.6.1) to $K$, and using Frobenius reciprocity, we get

$$
\left.\bar{X}\left(\rho / 2,-w_{i} \rho / 2\right)\right|_{K}=\operatorname{Ind}_{U}^{K}\left[F_{1}(\rho / 2) \otimes F_{2}\left(-w_{i} \rho / 2\right)\right],
$$

where $F_{1,2}$ are finite dimensional representations of the two factors $\operatorname{Spin}(2 p, \mathbb{C}) \times$ $\operatorname{Spin}(2 p, \mathbb{C})$ with infinitesimal character $\rho / 2$ and $-w_{i} \rho / 2$, respectively. The terms $\left[F_{1}(\rho / 2) \otimes F_{2}\left(-w_{i} \rho / 2\right)\right]$ are

(i) $V(1 / 2, \ldots, 1 / 2) \otimes V(1 / 2, \ldots, 1 / 2) \otimes V(0, \ldots, 0) \otimes V(0, \ldots, 0)$,

(ii) $V(1 / 2, \ldots,-1 / 2) \otimes V(1 / 2, \ldots, 1 / 2) \otimes V(0, \ldots, 0) \otimes V(0, \ldots, 0)$,

(iii) $V(1 / 2, \ldots, 1 / 2) \otimes V(0, \ldots, 0) \otimes V(0, \ldots, 0) \otimes V(1 / 2, \ldots, 1 / 2)$,

(iv) $V(1 / 2, \ldots, 1 / 2) \otimes V(0, \ldots, 0) \otimes V(0, \ldots, 0) \otimes V(1 / 2, \ldots,-1 / 2)$

as $\operatorname{Spin}(n) \times \operatorname{Spin}(n)$-representations (see Subsection 4.4 for the notation).

Lemma 4.7. Let $S P I N_{+}=V\left(\frac{1}{2}, \ldots, \frac{1}{2}\right)$ and $S P I N_{-}=V\left(\frac{1}{2}, \ldots, \frac{1}{2},-\frac{1}{2}\right) \in \widehat{\operatorname{Spin}(n)}$. Then

$$
\begin{aligned}
& S P I N_{+} \otimes S P I N_{+}=\bigoplus_{0 \leq k \leq\left[\frac{p}{2}\right]} V(\underbrace{1 \ldots 1}_{2 k}, \underbrace{0 \ldots 0}_{p-2 k}), \\
& S P I N_{+} \otimes S P I N_{-}=\bigoplus_{0 \leq k \leq\left[\frac{p-1}{2}\right]} V(\underbrace{1 \ldots 1}_{2 k+1} \underbrace{0 \ldots 0}_{p-2 k-1}) .
\end{aligned}
$$

Proof. The proof is straightforward.

Lemma 4.7 implies that (4.6.2) becomes

(i) $\left.\bar{X}(\rho / 2,-\rho / 2)\right|_{K} \quad=\operatorname{Ind}_{U}^{K}\left[\bigoplus_{0 \leq k \leq\left[\frac{p}{2}\right]} V(\underbrace{1, \ldots, 1}_{2 k}, 0, \ldots, 0) \otimes V(0, \ldots, 0)\right]$,

(ii) $\left.\bar{X}\left(\rho / 2,-w_{1} \rho / 2\right)\right|_{K}=\operatorname{Ind}_{U}^{K}\left[\bigoplus_{0 \leq k \leq\left[\frac{p-1}{2}\right]} V(\underbrace{1, \ldots, 1}_{2 k+1}, 0, \ldots, 0) \otimes V(0, \ldots, 0)\right]$,

(iii) $\left.\bar{X}\left(\rho / 2,-w_{2} \rho / 2\right)\right|_{K}=\operatorname{Ind}_{U}^{K}[V(1 / 2, \ldots, 1 / 2) \otimes V(1 / 2, \ldots, 1 / 2)]$,

(iv) $\left.\bar{X}\left(\rho / 2,-w_{3} \rho / 2\right)\right|_{K}=\operatorname{Ind}_{U}^{K}[V(1 / 2, \ldots, 1 / 2) \otimes V(1 / 2, \ldots,-1 / 2)]$. 


\section{Proposition 4.8.}

$(4.8 .1)$

$\left.\bar{X}(\rho / 2,-\rho / 2)\right|_{K}=\bigoplus V\left(a_{1}, \ldots, a_{n}\right), \quad$ with $a_{i} \in \mathbb{Z}, \quad \sum a_{i} \in 2 \mathbb{Z}$,

$\left.\bar{X}\left(\rho / 2,-w_{1} \rho / 2\right)\right|_{K}=\bigoplus V\left(a_{1}, \ldots, a_{n}\right), \quad$ with $a_{i} \in \mathbb{Z}, \quad \sum a_{i} \in 2 \mathbb{Z}+1$,

$\left.\bar{X}\left(\rho / 2,-w_{2} \rho / 2\right)\right|_{K}=\bigoplus V\left(a_{1}, \ldots, a_{n}\right), \quad$ with $a_{i} \in \mathbb{Z}+1 / 2, \quad \sum a_{i} \in 2 \mathbb{Z}+p$,

$\left.\bar{X}\left(\rho / 2,-w_{3} \rho / 2\right)\right|_{K}=\bigoplus V\left(a_{1}, \ldots, a_{n}\right), \quad$ with $a_{i} \in \mathbb{Z}+1 / 2, \quad \sum a_{i} \in 2 \mathbb{Z}+p+1$.

Proof. In the first two cases we can substitute $\left(G^{\text {split }}, K^{\text {split }}\right):=(\mathrm{SO}(2 p, 2 p), S[O(2 p)$ $\times O(2 p)])$ for $(K, U)$, and $(\operatorname{Spin}(2 p, 2 p), \operatorname{Spin}(2 p) \times \operatorname{Spin}(2 p) /\{ \pm(I, I)\})$ for the last two cases. The problem of computing the $K$-structure of $\bar{X}$ reduces to finding the finite-dimensional representations of $G^{\text {split }}$ which contain factors of $F(\rho / 2) \otimes$ $F\left(-w_{i} \rho / 2\right)$. Any finite-dimensional representation of $G^{\text {split }}$ is a Langlands quotient of a principal series. Principal series have fine lowest $K$-types (see Vog81). Let $M A$ be a split Cartan subgroup of $G^{\text {split }}$. A principal series is parametrized by a $(\delta, \nu) \in \widehat{M} A$. The $\delta$ are called fine, and each fine $K^{\text {split }}$-type $\mu$ is a direct sum of a Weyl group orbit of a fine $\delta$. This implies that the multiplicities in (4.7.2) are all one, and all the finite-dimensional representations occur in (i),(ii),(iii),(iv). The four formulas correspond to the various orbits of the $\delta$.

Case 2: $\mathcal{O}=\left[\begin{array}{lll}3 & 2^{2 k} & 1^{2 n-4 k-3}\end{array}\right], 0 \leq k \leq p-1$. Recall that

$$
\lambda_{\mathcal{O}}=\left(k+\frac{1}{2}, \ldots, \frac{3}{2}, \frac{1}{2} \mid n-k-2, \ldots, 1,0\right),
$$

and the integral system is $D_{k+1} \times D_{n-k-1}$. The irreducible modules are of the form $\bar{X}\left(\lambda_{L},-w \lambda_{R}\right)$ such that $\lambda_{\mathcal{O}}$ is dominant, $w_{i} \lambda_{\mathcal{O}}$ is antidominant for $D_{k+1} \times D_{n-k-1}$, and they factor to $\mathrm{SO}(2 n, \mathbb{C})$. These representations are listed in Subsection 4.4 .

We need to work with the real form $(\mathrm{SO}(r, s), S[O(r) \times O(s)])$. A representation of $O(n), r=2 m+\eta$ with $\eta=0$ or 1 , will be denoted by $V\left(a_{1}, \ldots, a_{m} ; \epsilon\right)$, with $\epsilon= \pm 1,1 / 2$ according to Weyl's convention, and $a_{1} \geq a_{2} \geq \cdots \geq a_{m} \geq 0$. If $a_{m}=0$, there are two inequivalent representations with this highest weight, one for $\epsilon=1$ and one for $\epsilon=-1$. Each restricts irreducibly to $\mathrm{SO}(r)$ as the representation $V\left(a_{1}, \ldots, a_{m}\right) \in \widehat{\mathrm{SO}(r)}$. When $a_{m} \neq 0$, there is a unique representation with this highest weight, $\epsilon=1 / 2$, or $\epsilon$ is suppressed altogether. The restriction of this representation to $\mathrm{SO}(r)$ is a sum of two representations, $V\left(a_{1}, \ldots, a_{m}\right)$ and $V\left(a_{1}, \ldots, a_{m-1},-a_{m}\right)$.

Representations of $\operatorname{Pin}(s)$ are parametrized in the same way, with $a_{1} \geq \cdots \geq$ $a_{m} \geq 0$ allowed to be nonnegative decreasing half-integers.

Representations of $S[O(r) \times O(s)]$ are parametrized by restrictions of $V\left(a ; \epsilon_{1}\right) \otimes$ $V\left(b ; \epsilon_{2}\right)$ with the following equivalences:

(1) If one of $\epsilon_{i}=\frac{1}{2}$, say, $\epsilon_{1}=\frac{1}{2}$, then $V\left(a ; \epsilon_{1}\right) \otimes V\left(b ; \epsilon_{2}\right)=V\left(a^{\prime} ; \delta_{1}\right) \otimes V\left(b^{\prime} ; \delta_{2}\right)$ if and only if $a=a^{\prime}, b=b^{\prime}, \epsilon_{1}=\delta_{1}, \epsilon_{2}=\delta_{2}$.

(2) If $\epsilon_{1}, \epsilon_{2}, \delta_{1}, \delta_{2} \in\{ \pm 1\}$, then $V\left(a ; \epsilon_{1}\right) \otimes V\left(b ; \epsilon_{2}\right)=V\left(a^{\prime} ; \delta_{1}\right) \otimes V\left(b^{\prime} ; \delta_{2}\right)$ iff $a=a^{\prime}, b=b^{\prime}, \epsilon_{1} \epsilon_{2}=\delta_{1} \delta_{2}$. 
Lemma 4.9. Let $P I N=V\left(\frac{1}{2} \ldots, \frac{1}{2}\right) \in \widehat{\operatorname{Pin}(s)}, s=2 m+\eta$ with $\eta=0$ or 1 . Then

$$
P I N \otimes P I N=\sum_{\ell=0}^{m-1} V(\underbrace{1 \ldots 1}_{\ell}, \underbrace{0 \ldots 0}_{m-\ell} ; \epsilon)+V(1, \ldots, 1 ; 1 / 2),
$$

where the sum is over $\epsilon=1$ and -1 .

Proof. Omitted.

We will use the groups $\underline{U}=S[O(2 k+2) \times O(2 n-2 k-2)] \subset \underline{K}=\mathrm{SO}(2 n)$. Again, the representations that we want are in Subsection 4.4 As before,

$$
\bar{X}\left(\lambda_{\mathcal{O}},-w_{i} \lambda_{\mathcal{O}}\right)=\sum_{w \in W\left(D_{k+1} \times D_{n-k-1}\right)} \epsilon(w) X\left(\lambda_{\mathcal{O}},-w w_{i} \lambda_{\mathcal{O}}\right) .
$$

Restricting to $K$, and using Frobenius reciprocity, (4.9.2) implies

$$
\left.\bar{X}\left(\lambda_{\mathcal{O}},-w_{i} \lambda_{\mathcal{O}}\right)\right|_{\underline{K}}=\operatorname{Ind} \frac{K}{\underline{K}}\left[F_{1}\left(\lambda_{\mathcal{O}}\right) \otimes F_{2}\left(-w_{i} \lambda_{\mathcal{O}}\right)\right] .
$$

The terms $\left[F_{1}\left(\lambda_{\mathcal{O}}\right) \otimes F_{2}\left(-w_{i} \lambda_{\mathcal{O}}\right)\right]$ are

(i) $V(1 / 2, \ldots, 1 / 2) \otimes V(0, \ldots, 0) \otimes V(1 / 2, \ldots, 1 / 2) \otimes V(0, \ldots, 0)$,

(ii) $V(1 / 2, \ldots, 1 / 2,-1 / 2) \otimes V(0, \ldots, 0) \otimes V(1 / 2, \ldots, 1 / 2,-1 / 2) \otimes V(0, \ldots, 0)$.

\section{Lemma 4.10.}

$(4.10 .1)$

$$
\begin{aligned}
\bar{X}\left(\lambda_{\mathcal{O}},-\lambda_{\mathcal{O}}\right)=\operatorname{Ind} \frac{K}{\underline{U}}[ & \sum_{0 \leq 2 \ell \leq k+1} V(\underbrace{1, \ldots, 1}_{2 \ell}, 0, \ldots, 0 ; 1) \otimes V(0, \ldots, 0 ; 1) \\
& \left.+\sum_{0 \leq 2 \ell \leq k+1} V(\underbrace{1, \ldots, 1}_{2 \ell}, 0, \ldots, 0 ; 1) \otimes V(0, \ldots, 0 ;-1)\right], \\
\bar{X}\left(\lambda_{\mathcal{O}},-w_{1} \lambda_{\mathcal{O}}\right)=\operatorname{Ind} \frac{K}{\underline{U}}[ & \sum_{0 \leq 2 \ell+1 \leq k+1} V(\underbrace{1, \ldots, 1}_{2 l+1}, 0, \ldots, 0 ; 1) \otimes V(0, \ldots, 0 ; 1) \\
& \left.+\sum_{0 \leq 2 \ell+1 \leq k+1} V(\underbrace{1, \ldots, 1}_{2 \ell+1}, 0, \ldots, 0 ; 1) \otimes V(0, \ldots, 0 ;-1)\right] .
\end{aligned}
$$

Proof. This follows from Lemma 4.9

\section{Proposition 4.11.}

$$
\begin{aligned}
& \left.\bar{X}\left(\lambda_{\mathcal{O}},-\lambda_{\mathcal{O}}\right)\right|_{K}=\bigoplus V\left(a_{1}, \ldots, a_{k}, 0, \ldots, 0\right), \quad \text { with } a_{i} \in \mathbb{Z}, \sum a_{i} \in 2 \mathbb{Z}, \\
& \left.\bar{X}\left(\lambda_{\mathcal{O}},-w_{1} \lambda_{\mathcal{O}}\right)\right|_{K}=\bigoplus V\left(a_{1}, \ldots, a_{k}, 0, \ldots, 0\right), \quad \text { with } a_{i} \in \mathbb{Z} . \sum a_{i} \in 2 \mathbb{Z}+1 .
\end{aligned}
$$

Proof. The proof is almost identical to that of Proposition 4.8. When $k=p-1$, the group $G^{\text {split }}$ in the proof of Proposition 4.8 is replaced by $G^{q s}=\mathrm{SO}(2 p, 2 p+2)$ and $K^{\text {split }}$ is replaced by $K^{q s}=S[O(2 p) \times O(2 p+2)]$. When $k<p-1$, the group $G^{\text {split }}$ is replaced by $G^{k+1, n-k-1}=S O(2 k+2,2 n-2 k-2)$ and $K^{\text {split }}$ is replaced by $K^{k+1, n-k-1}=S[O(2 k+2) \times O(2 n-2 k-2)]$. We follow Vog81. The $K-$ types $\mu$ in (4.10.1) have $\mathfrak{q}\left(\lambda_{L}\right)$ as the $\theta$-stable parabolic $\mathfrak{q}=\mathfrak{l}+\mathfrak{u}$ determined by 
$\xi=(0, \ldots, 0 ; \underbrace{1, \ldots, 1}_{n-2 k-2}, 0 \ldots, 0)$. The Levi component is $S[O(2 k) \times O(2 k+2)]$. The resulting $\mu_{L}=\mu-2 \rho(\mathfrak{u} \cap \mathfrak{s})$ are fine $U \cap L$-types. A bottom layer argument reduces the proof to the quasisplit case $n=2 p+1$.

Cases 3, 4: We use the infinitesimal characters in 4.3 and the representations are from Subsection 4.4 again.

In Case $4, \mathcal{O}=\left[\begin{array}{ll}2^{2 k} & 1^{2 n-4 k}\end{array}\right]$ with $k<p$. There is a unique irreducible representation with associated support $\mathcal{O}$, and it is spherical. It is a special unipotent representation with character given by BV85].

When $n=2 p$ and $k=p$, there are two nilpotent orbits $\mathcal{O}_{I, I I}=\left[2^{n}\right]_{I, I I}$. The representations $\Xi_{I, I I}$ in Subsection 4.4 are spherical representations, one each for $\mathcal{O}_{I, I I}$ that are not genuine. The two representations are induced irreducibly from the trivial representation of the parabolic subgroups with Levi components $\operatorname{GL}(n)_{I, I I}$. On the other hand, the representations $\Xi_{I, I I}^{\prime}$ are induced irreducibly from the character $D e t^{1 / 2}$ of the parabolic subgroups with Levi components $\operatorname{GL}(n)_{I, I I}$. All of these are unitary.

Proposition 4.12. The $K$-types of these representations are:

Case 3: $\mathcal{O}_{I, I I}=\left[2^{2 p}\right]_{I, I I}$ :

$$
\begin{aligned}
\left.\Xi_{I}\right|_{K} & =\bigoplus V\left(a_{1}, a_{1}, a_{3}, a_{3}, \ldots, a_{n-1}, a_{n-1}\right), \text { with } a_{i} \in \mathbb{Z}, \\
\left.\Xi_{I}^{\prime}\right|_{K} & =\bigoplus V\left(a_{1}, a_{1}, a_{3}, a_{3}, \ldots, a_{n-1}, a_{n-1}\right), \text { with } a_{i} \in \mathbb{Z}+1 / 2, \\
\left.\Xi_{I I}\right|_{K} & =\bigoplus V\left(a_{1}, a_{1}, a_{3}, a_{3}, \ldots, a_{n-1},-a_{n-1}\right), \text { with } a_{i} \in \mathbb{Z}, \\
\left.\Xi_{I I}^{\prime}\right|_{K} & =\bigoplus V\left(a_{1}, a_{1}, a_{3}, a_{3}, \ldots, a_{n-1},-a_{n-1}\right), \text { with } a_{i} \in \mathbb{Z}+1 / 2,
\end{aligned}
$$

satisfying $a_{1} \geq a_{3} \geq \cdots \geq a_{n-1} \geq 0$

Case 4: $\mathcal{O}=\left[\begin{array}{ll}2^{2 k} & 1^{2 n-4 k}\end{array}\right], 0 \leq k<n / 2:$

$$
\left.\Xi\right|_{K}=\bigoplus V\left(a_{1}, a_{1}, \ldots, a_{k}, a_{k}, 0, \ldots, 0\right), \text { with } a_{i} \in \mathbb{Z},
$$

satisfying $a_{1} \geq a_{3} \geq \cdots \geq a_{k} \geq 0$.

Proof. These are well known. The cases $\left[2^{n}\right]_{I, I I}$ follow by Cartan and Helgason's theorem since $\left(D_{n}, A_{n-1}\right)$ is a symmetric pair (for the real form $\left.\mathrm{SO}^{*}(2 n)\right)$. They also follow by the method outlined below for the other cases.

For $2 k<n$, the methods outlined in BP15 combined with Bar17 give the answer; the representations are $\Theta$-lifts of the trivial representation of $\operatorname{Sp}(2 k, \mathbb{C})$. More precisely $\bar{X}\left(\lambda_{\mathcal{O}},-\lambda_{\mathcal{O}}\right)$ is $\Omega /[\mathfrak{s p}(2 k, \mathbb{C}) \Omega]$, where $\Omega$ is the oscillator representation for the pair $O(2 n, \mathbb{C}) \times \operatorname{Sp}(2 k, \mathbb{C})$. The $K$-structure can then be computed using seesaw pairs, namely $\Omega$ is also the oscillator representation for the pair $O(2 n) \times \operatorname{Sp}(4 k, \mathbb{R})$.

4.13. We resume the notation used in Section 3. Let $\left(G_{0}, K\right)=(\operatorname{Spin}(2 n, \mathbb{C})$, $\operatorname{Spin}(2 n, \mathbb{C}))$. By comparing Propositions 3.8, 3.12, 3.16 and the $K$-structure of representations listed in this section, we have the following matchup:

Case 1: $\left.\Xi_{i}\right|_{K}=R\left(\mathcal{O}, \psi_{i}\right), 1 \leq i \leq 4$;

Case 2: $\left.\Xi_{i}\right|_{K}=R\left(\mathcal{O}, \psi_{i}\right), i=1,2$;

Case 3: $\left.\Xi_{I}\right|_{K}=R\left(\mathcal{O}_{I}\right.$, Triv $),\left.\Xi_{I}^{\prime}\right|_{K}=R\left(\mathcal{O}_{I}, S g n\right)$,

$$
\left.\Xi_{I I}\right|_{K}=R\left(\mathcal{O}_{I I}, \text { Triv }\right),\left.\Xi_{I I}^{\prime}\right|_{K}=R\left(\mathcal{O}_{I I}, S g n\right) ;
$$

Case 4: $\left.\Xi\right|_{K}=R(\mathcal{O}$, Triv $)$. 
Then the following theorem follows.

Theorem 4.14. Attain the notation above. Let $G_{0}=\operatorname{Spin}(2 n, \mathbb{C})$ be viewed as a real group. The $K$-structure of each representations in $\mathcal{U}_{G_{0}}\left(\mathcal{O}, \lambda_{\mathcal{O}}\right)$ is calculated explicitly and matches the $K$-structure of the $R(\mathcal{O}, \psi)$ with $\psi \in \widehat{A_{K}(\mathcal{O})}$. That is, there is a 1-1 correspondence $\psi \in \widehat{A_{K}(\mathcal{O})} \longleftrightarrow \Xi(\mathcal{O}, \psi) \in \mathcal{U}_{G_{0}}\left(\mathcal{O}, \lambda_{\mathcal{O}}\right)$ satisfying

$$
\left.\Xi(\mathcal{O}, \psi)\right|_{K} \cong R(\mathcal{O}, \psi) \text {. }
$$

\section{Clifford Algebras AND Spin groups}

Since the main interest is in the case of $\operatorname{Spin}(V)$, the simply connected groups of type $D$, we realize everything in the context of the Clifford algebra.

5.1. Let $(V, Q)$ be a quadratic space of even dimension $2 n$, with a basis $\left\{e_{i}, f_{i}\right\}$ with $1 \leq i \leq n$, satisfying $Q\left(e_{i}, f_{j}\right)=\delta_{i j}, Q\left(e_{i}, e_{j}\right)=Q\left(f_{i}, f_{j}\right)=0$. Occasionally we will replace $e_{j}, f_{j}$ by two orthogonal vectors $v_{j}, w_{j}$ satisfying $Q\left(v_{j}, v_{j}\right)=Q\left(w_{j}, w_{j}\right)=1$, and orthogonal to the $e_{i}, f_{i}$ for $i \neq j$. Precisely they will satisfy $v_{j}=\left(e_{j}+f_{j}\right) / \sqrt{2}$ and $w_{j}=\left(e_{j}-f_{j}\right) /(i \sqrt{2})$ (where $i:=\sqrt{-1}$, not an index). Let $C(V)$ be the Clifford algebra with automorphisms $\alpha$ defined by $\alpha\left(x_{1} \cdots x_{r}\right)=(-1)^{r} x_{1} \cdots x_{r}$ and $\star$ given by $\left(x_{1} \cdots x_{r}\right)^{\star}=(-1)^{r} x_{r} \cdots x_{1}$, subject to the relation $x y+y x=2 Q(x, y)$ for $x, y \in V$. The double cover of $O(V)$ is

$$
\operatorname{Pin}(V):=\left\{x \in C(V) \mid x \cdot x^{\star}=1, \alpha(x) V x^{\star} \subset V\right\} .
$$

The double cover $\operatorname{Spin}(V)$ of $\mathrm{SO}(V)$ is given by the elements in $\operatorname{Pin}(V)$ which are in $C(V)^{\text {even }}$, i.e., $\operatorname{Spin}(V):=\operatorname{Pin}(V) \cap C(V)^{\text {even }}$. For Spin, $\alpha$ can be suppressed from the notation since it is the identity.

The action of $\operatorname{Pin}(V)$ on $V$ is given by $\rho(x) v=\alpha(x) v x^{\star}$. The element $-I \in$ $\mathrm{SO}(V)$ is covered by

$$
\pm \mathcal{E}_{2 n}= \pm i^{n-1} v_{n} w_{n} \prod_{1 \leq j \leq n-1}\left[1-e_{j} f_{j}\right]= \pm i^{n} \prod_{1 \leq j \leq n}\left[1-e_{j} f_{j}\right] .
$$

These elements satisfy

The center of $\operatorname{Spin}(V)$ is

$$
\mathcal{E}_{2 n}^{2}= \begin{cases}+I d & \text { if } n \in 2 \mathbb{Z} \\ -I d & \text { otherwise }\end{cases}
$$

$$
Z(\operatorname{Spin}(V))=\left\{ \pm I, \pm \mathcal{E}_{2 n}\right\} \cong \begin{cases}\mathbb{Z}_{2} \times \mathbb{Z}_{2} & \text { if } n \text { is even } \\ \mathbb{Z}_{4} & \text { if } n \text { is odd }\end{cases}
$$

The Lie algebra of $\operatorname{Pin}(V)$ as well as $\operatorname{Spin}(V)$ is formed of elements of even order $\leq 2$ satisfying

$$
x+x^{\star}=0 .
$$

The adjoint action is ad $x(y)=x y-y x$. A Cartan subalgebra and the root vectors corresponding to the usual basis in Weyl normal form are formed of the elements

$$
\begin{array}{ll}
\left(1-e_{i} f_{i}\right) / 2 & \longleftrightarrow H\left(\epsilon_{i}\right), \\
e_{i} e_{j} / 2 & \longleftrightarrow X\left(-\epsilon_{i}-\epsilon_{j}\right), \\
e_{i} f_{j} / 2 & \longleftrightarrow X\left(-\epsilon_{i}+\epsilon_{j}\right), \\
f_{i} f_{j} / 2 & \longleftrightarrow X\left(\epsilon_{i}+\epsilon_{j}\right) .
\end{array}
$$


5.2. Nilpotent orbits. We write $\widetilde{K}=\operatorname{Spin}(V)=\operatorname{Spin}(2 n, \mathbb{C})$ and $K=\operatorname{SO}(V)=$ $\mathrm{SO}(2 n, \mathbb{C})$. A nilpotent orbit of an element $e$ will have Jordan blocks denoted by $(5.2 .1)$

$$
\begin{aligned}
& e_{1} \longrightarrow e_{2} \longrightarrow \cdots \longrightarrow e_{k} \longrightarrow v \longrightarrow-f_{k} \longrightarrow f_{k-1} \longrightarrow-f_{k-2} \longrightarrow \cdots \longrightarrow \pm f_{1} \longrightarrow 0 \text {, } \\
& e_{1} \longrightarrow e_{2} \quad \longrightarrow \quad \longrightarrow \quad e_{2 \ell} \longrightarrow 0, \\
& f_{2 \ell} \longrightarrow-f_{2 \ell-1} \longrightarrow \ldots \longrightarrow-f_{1} \longrightarrow 0
\end{aligned}
$$

with the conventions about the $e_{i}, f_{j}, v$ (equal to some appropriate $v_{m}$ ) as before. Every block is realized by a representative $E$, with the arrow in the block standing for the $\operatorname{map} \operatorname{ad}_{E}$. More precisely, a realization of the odd block is given by the representative $\frac{1}{2}\left(\sum_{i=1}^{k-1} e_{i+1} f_{i}+v f_{k}\right)$, and a realization of the even blocks by $\frac{1}{2}\left(\sum_{i=1}^{2 \ell-1} e_{i+1} f_{i}\right)$. When there are only even blocks, there are two orbits; one block of the form $\frac{1}{2}\left(\sum_{i=1}^{2 \ell-1} e_{i+1} f_{i}+e_{2 \ell} f_{2 \ell-1}\right)$ is replaced by $\frac{1}{2}\left(\sum_{i=1}^{2 \ell-1} e_{i+1} f_{i}+f_{2 \ell} f_{2 \ell-1}\right)$. Since the sizes of all blocks sum up to $2 n$, there is an even number of odd sized blocks; any two blocks of equal odd size $2 k+1$ can be replaced by a pair of blocks of the same form as the even ones.

The centralizer of $e$ in $\mathfrak{s o}(V)$ has a Levi component isomorphic to a product of $\mathfrak{s o}\left(r_{2 k+1}\right)$ and $\mathfrak{s p}\left(2 r_{2 \ell}\right)$, where $r_{j}$ is the number of blocks of size $j$. The centralizer of $e$ in $\mathrm{SO}(V)$ has Levi component $\prod \mathrm{Sp}\left(2 r_{2 \ell}\right) \times S\left[\prod O\left(r_{2 k+1}\right)\right]$. For each odd sized block define

$$
\mathcal{E}_{2 k+1}=i^{k} v \prod\left(1-e_{j} f_{j}\right)
$$

This is an element in $\operatorname{Pin}(V)$, and acts by $-I d$ on the block. Even products of $\pm \mathcal{E}_{2 k+1}$ belong to $\operatorname{Spin}(V)$ and represent the connected components of $C_{\widetilde{K}}(e)$.

Proposition 5.3. Let $m$ be the number of distinct odd blocks. Then

$$
A_{K}(\mathcal{O}) \cong \begin{cases}\mathbb{Z}_{2}^{m-1} & \text { if } m>0, \\ 1 & \text { if } m=0 .\end{cases}
$$

Furthermore,

(1) If e has an odd block of size $2 k+1$ with $r_{2 k+1}>1$, then $A_{\widetilde{K}}(\mathcal{O}) \cong A_{K}(\mathcal{O})$.

(2) If all $r_{2 k+1} \leq 1$, then there is an exact sequence

$$
1 \longrightarrow\{ \pm I\} \longrightarrow A_{\widetilde{K}}(\mathcal{O}) \longrightarrow A_{K}(\mathcal{O}) \longrightarrow 0 .
$$

Proof. Assume that there is an $r_{2 k+1}>1$. Let

$$
\begin{array}{ccccc}
e_{1} & \rightarrow \ldots & \rightarrow & e_{2 k+1} & \rightarrow 0, \\
f_{2 k+1} & \rightarrow \ldots & \rightarrow & -f_{1} & \rightarrow 0
\end{array}
$$

be two of the blocks. In the Clifford algebra this element is $e=\left(e_{2} f_{1}+\cdots+\right.$ $\left.e_{2 k+1} f_{2 k}\right) / 2$. The element $\sum_{j=1}^{2 k+1}\left(1-e_{j} f_{j}\right)$ in the Lie algebra commutes with $e$. So its exponential

$$
\prod \exp \left(i \theta\left(1-e_{j} f_{j}\right) / 2\right)=\prod\left[\cos \theta / 2+i \sin \theta / 2\left(1-e_{j} f_{j}\right)\right]
$$

also commutes with $e$. At $\theta=0$, the element in (5.3.1) is $I$; at $\theta=2 \pi$, it is $-I$. Thus $-I$ is in the connected component of the identity of $A_{\widetilde{K}}(\mathcal{O})\left(\right.$ when $r_{2 k+1}>1$ ), and therefore $A_{\widetilde{K}}(\mathcal{O})=A_{K}(\mathcal{O})$.

Assume there are no blocks of odd size. Then $C_{K}(\mathcal{O}) \cong \prod \operatorname{Sp}\left(r_{2 l}\right)$ is simply connected, so $C_{\widetilde{K}}(\mathcal{O}) \cong C_{K}(\mathcal{O}) \times\{ \pm I\}$. Therefore $A_{\widetilde{K}}(\mathcal{O}) \cong \mathbb{Z}_{2}$. 
Assume there are $m$ distinct odd blocks with $m \in 2 \mathbb{Z}_{>0}$ and $r_{2 k_{1}+1}=\cdots=$ $r_{2 k_{m}+1}=1$. In this case, $C_{K}(\mathcal{O}) \cong \prod \mathrm{Sp}\left(r_{2 l}\right) \times S[\underbrace{O(1) \times \cdots \times O(1)}_{m}]$, and hence $A_{K}(\mathcal{O}) \cong \mathbb{Z}_{2}^{m-1}$. Even products of $\left\{ \pm \mathcal{E}_{2 k_{j}+1}\right\}$ are representatives of elements in $A_{\widetilde{K}}(\mathcal{O})$. They satisfy

$$
\mathcal{E}_{2 k+1} \cdot \mathcal{E}_{2 \ell+1}= \begin{cases}-\mathcal{E}_{2 \ell+1} \cdot \mathcal{E}_{2 k+1} & k \neq \ell \\ (-1)^{k} I & k=\ell\end{cases}
$$

\section{Corollary 5.4.}

(1) If $\mathcal{O}=\left[\begin{array}{ll}32^{n-2} & 1]\end{array}\right]$ then $A_{\widetilde{K}}(\mathcal{O}) \cong \mathbb{Z}_{2} \times \mathbb{Z}_{2}=\left\{ \pm \mathcal{E}_{3} \cdot \mathcal{E}_{1}, \pm I\right\}$.

(2) If $\mathcal{O}=\left[\begin{array}{lll}3 & 2^{2 k} & 1^{2 n-4 k-3}\end{array}\right]$ with $2 n-4 k-3>1$, then $A_{\widetilde{K}}(\mathcal{O}) \cong \mathbb{Z}_{2}$.

(3) If $\mathcal{O}=\left[2^{n}\right]_{I, I I}$ ( $n$ even), then $A_{\widetilde{K}}(\mathcal{O}) \cong \mathbb{Z}_{2}$.

(4) If $\mathcal{O}=\left[2^{2 k} 1^{2 n-4 k}\right]$ with $2 k<n$, then $A_{\widetilde{K}}(\mathcal{O}) \cong 1$.

In all cases $C_{\widetilde{K}}(\mathcal{O})=Z(\widetilde{K}) \cdot C_{\widetilde{K}}(\mathcal{O})^{0}$.

\section{References}

[ABV92] Jeffrey Adams, Dan Barbasch, and David A. Vogan Jr., The Langlands classification and irreducible characters for real reductive groups, Progress in Mathematics, vol. 104, Birkhäuser Boston, Inc., Boston, MA, 1992. MR:1162533

[AHV98] Jeffrey Adams, Jing-Song Huang, and David A. Vogan Jr., Functions on the model orbit in $E_{8}$, Represent. Theory 2 (1998), 224-263, DOI 10.1090/S1088-4165-98-00048X. MR.1628031

[Bar17] Dan Barbasch, Unipotent representations and the dual pair correspondence, Representation theory, number theory, and invariant theory, Progr. Math., vol. 323, Birkhäuser/Springer, Cham, 2017, pp. 47-85. MR3753908

[Bar89] Dan Barbasch, The unitary dual for complex classical Lie groups, Invent. Math. 96 (1989), no. 1, 103-176, DOI 10.1007/BF01393972. MR981739

[BP11] Dan Barbasch and Pavle Pandžić, Dirac cohomology and unipotent representations of complex groups, Noncommutative geometry and global analysis, Contemp. Math., vol. 546, Amer. Math. Soc., Providence, RI, 2011, pp. 1-22, DOI 10.1090/conm/546/10782. MR2815128

[BP15] Dan Barbasch and Pavle Pandžić, Dirac cohomology of unipotent representations of $S p(2 n, \mathbb{R})$ and $U(p, q)$, J. Lie Theory 25 (2015), no. 1, 185-213. MR3345832

[Bre99] Alfredo O. Brega, On the unitary dual of $\operatorname{Spin}(2 n, \mathbf{C})$, Trans. Amer. Math. Soc. 351 (1999), no. 1, 403-415, DOI 10.1090/S0002-9947-99-02173-X. MR.1473432

[BV85] Dan Barbasch and David A. Vogan Jr., Unipotent representations of complex semisimple groups, Ann. of Math. (2) 121 (1985), no. 1, 41-110, DOI 10.2307/1971193. MR782556

[BT18] Dan Barbasch and Wan-Yu Tsai, Representations associated to small niltpotent orbits for real spin groups, J. Lie Theory 28 (2018), no. 4, 987-1042. MR3831429

[CM93] David H. Collingwood and William M. McGovern, Nilpotent orbits in semisimple Lie algebras, Van Nostrand Reinhold Mathematics Series, Van Nostrand Reinhold Co., New York, 1993. MR1251060

[Hum08] James E. Humphreys, Representations of semisimple Lie algebras in the BGG category $\mathcal{O}$, Graduate Studies in Mathematics, vol. 94, American Mathematical Society, Providence, RI, 2008. MR2428237

[Kin04] Donald R. King, Classification of spherical nilpotent orbits in complex symmetric space, J. Lie Theory 14 (2004), no. 2, 339-370. MR2066860

[KP82] Hanspeter Kraft and Claudio Procesi, On the geometry of conjugacy classes in classical groups, Comment. Math. Helv. 57 (1982), no. 4, 539-602, DOI 10.1007/BF02565876. MR694606 
[KV95] Anthony W. Knapp and David A. Vogan Jr., Cohomological induction and unitary representations, Princeton Mathematical Series, vol. 45, Princeton University Press, Princeton, NJ, 1995. MR1330919

[McG94] William M. McGovern, Rings of regular functions on nilpotent orbits. II. Model algebras and orbits, Comm. Algebra 22 (1994), no. 3, 765-772, DOI 10.1080/00927879408824874. MR.1261003

[Pan94] Dmitrii I. Panyushev, Complexity and nilpotent orbits, Manuscripta Math. 83 (1994), no. 3-4, 223-237, DOI 10.1007/BF02567611. MR.1277527

[Vog81] David A. Vogan Jr., Representations of real reductive Lie groups, Progress in Mathematics, vol. 15, Birkhäuser, Boston, Mass., 1981. MR632407

[Vog00] David A. Vogan Jr., The method of coadjoint orbits for real reductive groups, Representation theory of Lie groups (Park City, UT, 1998), IAS/Park City Math. Ser., vol. 8, Amer. Math. Soc., Providence, RI, 2000, pp. 179-238. MR 1737729

[Vog91] David A. Vogan Jr., Associated varieties and unipotent representations, Harmonic analysis on reductive groups (Brunswick, ME, 1989), Progr. Math., vol. 101, Birkhäuser Boston, Boston, MA, 1991, pp. 315-388. MR.1168491

[War72] Garth Warner, Harmonic analysis on semi-simple Lie groups. I: Die Grundlehren der mathematischen Wissenschaften, Band 188, Springer-Verlag, New York-Heidelberg, 1972. MR0498999

Department of Mathematics, Cornell University, IthacA, New York 14850

Email address: barbasch@math.cornell.edu

Institute of Mathematics, ACAdemia Sinica, 6F, Astronomy-Mathematics Building, No. 1, Sec. 4, Roosevelt Road, Taipei 10617, Taiwan

Current address: Department of Mathematics and Statistics, University of Ottawa, Ontario, Canada

Email address: wtsai@uottawa.ca, wanyupattsai@gmail.com 